\title{
The fermion propagator in cosmological spaces with constant deceleration
}

\author{
Jurjen F Koksma and Tomislav Prokopec \\ Institute for Theoretical Physics (ITP) and Spinoza Institute, Utrecht University, Postbus 80195, \\ 3508 TD Utrecht, The Netherlands \\ E-mail: J.F.Koksma@uu.nl and T.Prokopec@uu.nl
}

Received 3 February 2009, in final form 14 April 2009

Published 14 May 2009

Online at stacks.iop.org/CQG/26/125003

\begin{abstract}
We calculate the fermion propagator in Friedmann-Lemaitre-RobertsonWalker (FLRW) spacetimes with constant deceleration $q=\epsilon-1, \epsilon=-\dot{H} / H^{2}$ for excited states. For fermions whose mass is generated by a scalar field through a Yukawa coupling $m=g_{\mathrm{Y}} \phi$, we assume $\phi \propto H$. We first solve the mode functions by splitting the spinor into a direct product of helicity and chirality spinors. We also allow for non-vacuum states. We normalize the spinors using a consistent canonical quantization and by requiring orthogonality of particle and anti-particle spinors. We apply our propagator to calculate the one-loop effective action and renormalize using dimensional regularization. Since the Hubble parameter is now treated dynamically, this paves the way to study the dynamical backreaction of fermions on the background spacetime.
\end{abstract}

PACS numbers: 98.80.-k, 04.62.+v, 03.70.+k

\section{Introduction}

Partly motivated by our increased capability in recent years to perform accurate observations on the sky, we have turned our attention to investigating the impact of quantum effects on the evolution of the universe. In pursuit of this goal, quantum field theory in cosmological spacetimes continues to be an increasingly important field of research. Among other things, it investigates how quantum fluctuations affect the background spacetime in perturbative quantum gravity, a process also known as quantum backreaction.

A line of research deals, for example, with the backreaction of quantum fields whose spectrum is nearly flat. Examples of these fields are the minimally coupled massless scalar and the graviton. Consequently, these fields are expected to yield a substantial backreaction [1-14]. Also, fermions in curved spacetimes and, in particular, maximally symmetric spacetimes were investigated (see, e.g., [15-19]). 
Another approach to this question is concerned with calculating the possible effect of the trace anomaly on the background spacetime. Some authors argue that this effect, particularly in relation to the cosmological constant problem, could be significant [20-25]; however, also see [26].

An essential element to study quantum effects in curved spaces is the propagator. Due to its high degree of symmetry, de Sitter spacetime proves to be ideally suited for calculating various quantum effects. Moreover, results are immediately applicable to inflation, cosmologists' favourite paradigm for a brief exponentially fast expansion in the early universe. Chernikov and Tagirov calculated the scalar propagator in de Sitter spacetime [27]; also see [28, 29]. The vector propagator was constructed by [30, 31] and the graviton propagator received contributions from [2, 3, 32-35]. Candelas and Raine calculated the fermion propagator [15].

However, de Sitter spacetime suffers from several drawbacks. Firstly, pure de Sitter spacetime is never realized in nature. Since de Sitter spacetime corresponds to a globally constant Hubble parameter $H$, this is in reality never attained. Secondly, de Sitter spacetime is non-dynamical. It is therefore inconsistent to study backreaction effects while at the same time assuming a constant background. We should allow the background to change to encompass all possible backreaction effects consistently. Therefore, it is necessary to consider propagators in more general spacetimes.

The scalar and graviton propagators in quasi de Sitter spacetime were calculated by [11] and subsequently generalized to Friedmann-Lemaître-Robertson-Walker or FLRW spacetimes with constant deceleration by [12-14]. In this paper, we calculate the fermion propagator in FLRW spacetimes with constant deceleration.

We should touch upon an important issue. In the massive case, we need two additional constraints to be satisfied in order to solve for the propagator:

$$
\begin{aligned}
& \epsilon \equiv-\frac{\dot{H}}{H^{2}}=\mathrm{const} \\
& \frac{m}{H}=\text { const. }
\end{aligned}
$$

Recall that all interesting epochs in the evolution of our universe satisfy the first constraint. In the matter era we have $\epsilon=3 / 2$, in the radiation-dominated epoch we find $\epsilon=2$. It also serves as an approximation to both inflation and the current dark-energy-dominated epoch when $\epsilon \ll 1$. Note that $\epsilon$ coincides with the slow-roll parameter of inflation and it is straightforwardly related to the somewhat more familiar deceleration parameter $q=\epsilon-1$. Hence $(1 a)$ is equivalent to requiring a constant deceleration parameter.

The second assumption $(1 b)$ is required to analytically derive the propagator in the massive case. In Yukawa theory, the mass of the fermion is generated by a scalar field for which we can achieve $\phi \propto H$ in several cases to which we will turn shortly.

We then calculate the one-loop effective action induced by fermions using our propagator. The one-loop backreaction arises from integrating out a free, quadratic fermion field and, using dimensional regularization, this generates a correction to the (classical) Friedmann equations.

The Hubble parameter occurring in this effective action is now a dynamical quantity. Consequently, the backreaction of these fermions on the background spacetime can thus be analysed dynamically. Also, when the fermions are coupled to a scalar field, this opens up the possibility of studying the impact of the fermions on the evolution of scalar fields.

This paper is outlined as follows. In section 2, we review the basic theory required to study fermions in curved spaces and we establish our notation. Sections 3 and 4 are devoted to deriving the fermionic propagator in FLRW spacetimes in the massless and massive cases, 
respectively. Finally, in section 5 we apply our propagator to calculate the one-loop effective action.

\section{Fermions in FLRW spacetimes}

\subsection{The Dirac equation}

Fermions are in general $D$-dimensional curved spacetimes described by the action [36]:

$$
S=\int \mathrm{d}^{D} x \sqrt{-g}\left\{\frac{\mathrm{i}}{2}\left[\bar{\psi} \gamma^{\mu} \nabla_{\mu} \psi-\left(\nabla_{\mu} \bar{\psi}\right) \gamma^{\mu} \psi\right]-m \bar{\psi} \psi\right\}
$$

where the Dirac matrices $\gamma^{\mu}$ satisfy the following anti-commutation relations:

$$
\left\{\gamma^{\mu}, \gamma^{\nu}\right\}=-2 g^{\mu \nu} \text {. }
$$

Variation with respect to $\bar{\psi}=\psi^{\dagger} \gamma^{0}$ yields the equation of motion the fermion field $\psi$ satisfies:

$$
\mathrm{i} \gamma^{\mu} \nabla_{\mu} \psi(x)-m \psi(x)=0 \text {. }
$$

We will make use of the vierbein formalism which can be thought of as a transformation of the metric tensor to a locally flat Minkowski metric:

$$
g_{\mu \nu}(x)=e_{\mu}^{a}(x) e_{\nu}^{b}(x) \eta_{a b},
$$

where $\eta_{a b}=\operatorname{diag}(-1,1, \ldots, 1)$ is the Minkowski metric. We use Greek letters to run over spacetime indices whereas we use Latin letters either for the tangent space indices or for spinor indices. It will be clear from the context which interpretation we are using. The Minkowski metric and the (flat-space) Dirac matrices $\gamma^{a}$ are spacetime independent. Spacetime and tangent space indices are raised or lowered by making use of the full metric or Minkowski metric, respectively. The covariant derivative acting on a Dirac spinor is defined as

$$
\nabla_{\mu} \psi(x)=\partial_{\mu} \psi(x)-\Gamma_{\mu} \psi(x),
$$

where the spin connection is given by

$$
\Gamma_{\mu}=-\frac{1}{8} e_{c}^{\nu}\left(\partial_{\mu} e_{\nu d}-\Gamma_{\mu \nu}^{\alpha} e_{\alpha d}\right)\left[\gamma^{c}, \gamma^{d}\right]
$$

such that $\nabla_{\mu} \gamma_{\nu}=0$. Let us specialize to flat FLRW spacetimes in which the metric is given by $g_{\mu \nu}=a^{2}(\eta) \eta_{\mu \nu}$. Here, $a(\eta)$ is the scale factor of the universe in conformal time defined by $\mathrm{d} t=a(\eta) \mathrm{d} \eta$. In FLRW spacetimes the vierbeins are a function of conformal time only:

$$
\begin{aligned}
& e_{\mu}^{b}(\eta)=\delta_{\mu}^{b} a(\eta), \\
& e_{b}^{\mu}(\eta)=\delta_{b}^{\mu} a^{-1}(\eta) .
\end{aligned}
$$

We thus find

$$
\mathrm{i} \gamma^{\mu} \nabla_{\mu} \psi(x)=a^{-\frac{D+1}{2}}(\eta) \mathrm{i} \gamma^{b} \partial_{b}\left(a^{\frac{D-1}{2}}(\eta) \psi(x)\right) .
$$

This useful identity relates covariant derivatives to partial derivatives in the tangent space.

Since we work in the chirality representation, the flat-space Dirac matrices are given by

$$
\gamma^{0}=\left(\begin{array}{ll}
0 & \mathbb{I} \\
\mathbb{I} & 0
\end{array}\right)
$$

where $\mathbb{I}$ is the $2 \times 2$ identity matrix and

$$
\gamma^{i}=\left(\begin{array}{cc}
0 & \sigma^{i} \\
-\sigma^{i} & 0
\end{array}\right),
$$

and where $\sigma^{i}$ denote the Pauli matrices. 


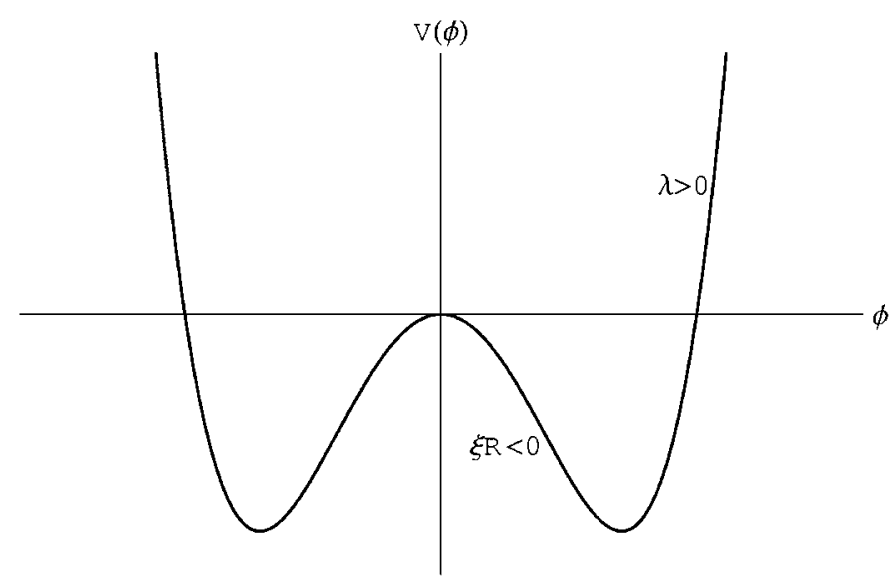

Figure 1. Qualitative sketch for the 'Mexican hat' potential (15) assuming $\mu^{2} \ll|\xi R|$. Near the minimum of the potential (16), assumption $(1 b)$ is satisfied.

In Yukawa theory, the mass of the fermion appearing in equation (2) is time dependent and generated by a scalar field. Let us thus briefly consider the complex system of a fermion whose mass is generated by a homogeneous scalar field $\phi(\eta)$, both of which are coupled to gravity. The action is

$$
\begin{aligned}
S=\int \mathrm{d}^{D} x \sqrt{-g} & \left\{-\frac{1}{2} g^{\mu \nu} \partial_{\mu} \phi \partial_{\nu} \phi-V(\phi)-g_{\mathrm{Y}} \bar{\psi} \psi \phi\right\} \\
+ & \int \mathrm{d}^{D} x \sqrt{-g}\left\{\frac{\mathrm{i}}{2}\left[\bar{\psi} \gamma^{\mu} \nabla_{\mu} \psi-\left(\nabla_{\mu} \bar{\psi}\right) \gamma^{\mu} \psi\right]\right\} .
\end{aligned}
$$

Here $g_{\mathrm{Y}}$ is the Yukawa coupling constant. By comparing equations (12) and (2) we can thus identify the mass of the fermion as

$$
m(\eta)=g_{\mathrm{Y}} \phi(\eta)
$$

Furthermore, the (classical) evolution of the homogeneous background field $\phi(\eta)$ is governed by

$$
\square \phi-\partial_{\phi} V(\phi, R)=0,
$$

where $V(\phi, R)$ is the potential:

$$
V(\phi, R)=\frac{\mu^{2}}{2} \phi^{2}+\frac{\xi}{2} R \phi^{2}+\frac{\lambda}{4 !} \phi^{4} .
$$

Here $\xi$ denotes some non-minimal coupling to gravity, and $R$ is the Ricci scalar. In FLRW spacetimes in $D=4$ we have $R=6\left(\dot{H}+2 H^{2}\right)=6(2-\epsilon) H^{2}$, where the Hubble parameter is given by $H=\dot{a} / a$. Dots denote derivatives with respect to cosmic time and dashes correspond to conformal time derivatives. Assuming $\mu^{2} \ll \xi R$, the solution of (14) is

$$
\phi= \pm H \sqrt{\frac{6}{\lambda}[\epsilon(3-2 \epsilon)-6 \xi(2-\epsilon)]} .
$$

Clearly, $\phi \propto H$ and assumption $(1 b)$ is satisfied. Moreover, observe that we need $\xi<\epsilon(3-2 \epsilon) /\{6(2-\epsilon)\}$. The potential is depicted in figure 1. Given a potential of the form (15), we identify some interpretations leading to this scenario. In the early universe, the Higgs field satisfies $\mu^{2} \ll|\xi R|$ and hence (16). A quintessence field has $\mu^{2}=0$ and (16) 
is also satisfied in the late universe. Finally, we can infer that a massless minimally coupled scalar field is also allowed in the regime where $0<\epsilon<3 / 2$ or $\epsilon>2$.

We work in the approximation where $\dot{\epsilon}=0$, which is not equivalent to the standard slow-roll approximation of inflation, where one introduces the slow-roll parameters $\epsilon$ and $\eta$. These parameters are allowed to vary slowly in time such that $\dot{\epsilon}$ and $\dot{\eta}$ are higher order in the slow-roll parameters and can be neglected when one is interested in the leading-order behaviour only. When $\epsilon$ is allowed to vary in time, equation (16) generalizes to $\phi=\alpha H$, where

$$
\alpha= \pm \sqrt{\frac{6}{\lambda}\left[\epsilon(3-2 \epsilon)+\frac{\dot{\epsilon}}{H}-6 \xi(2-\epsilon)\right]}+\mathcal{O}(\dot{\alpha}) .
$$

The leading-order slow-roll approximation to this equation corresponds to

$$
\alpha= \pm \sqrt{\frac{6}{\lambda}[3 \epsilon-6 \xi(2-\epsilon)]} .
$$

Note that the equation above depends on $\epsilon$, whereas it does not depend on $\eta$.

\subsection{The propagator}

Depending on which pole prescription one uses in the spirit of the Schwinger-Keldysh formalism (see, e.g., [37-39]), one can obtain different propagators such as the anti-timeordered, time-ordered or Wightman propagators. We are primarily interested in the timeordered or Feynman fermion propagator, defined by

$$
\begin{aligned}
\mathrm{i} S_{\mathrm{F}}^{a b}(x, \tilde{x}) & =\left\langle\Omega\left|\mathrm{T}\left\{\hat{\psi}_{a}(x) \hat{\bar{\psi}}_{b}(\tilde{x})\right\}\right| \Omega\right\rangle \\
& =\theta(\eta-\tilde{\eta})\left\langle\Omega\left|\hat{\psi}_{a}(x) \hat{\bar{\psi}}_{b}(\tilde{x})\right| \Omega\right\rangle-\theta(\tilde{\eta}-\eta)\left\langle\Omega\left|\hat{\bar{\psi}}_{b}(\tilde{x}) \hat{\psi}_{a}(x)\right| \Omega\right\rangle .
\end{aligned}
$$

The symbols $a$ and $b$ are the spinor indices, and $|\Omega\rangle$ denotes the state of the system. Also note the minus sign in front of the second $\theta$-function. The fermion propagator at tree level satisfies

$$
\sqrt{-g}\left[\mathrm{i} \gamma^{\mu} \nabla_{\mu}^{x}-m\right] \mathrm{i} S_{\mathrm{F}}^{a b}(x, \tilde{x})=\mathrm{i} \delta^{D}(x-\tilde{x}) \mathbb{I}^{a b},
$$

and a likewise equation when $x$ and $\tilde{x}$ are interchanged.

\subsection{Properties of FLRW spacetimes}

We have already discussed that FLRW spacetimes can be characterized by the scale factor $a$ as a function of either cosmic or conformal time. There are, however, a few more important properties of this spacetime that are worth mentioning here.

An important relation that we will use throughout this manuscript is

$$
a(\eta) \eta=-\frac{1}{H(\eta)(1-\epsilon)} .
$$

This equation is equivalent to assumption $(1 a)$; see $[12,13]$. Let us define some relevant geometrical functions:

$$
\begin{aligned}
y_{++}(x, \tilde{x}) & =\frac{\Delta x_{++}^{2}(x, \tilde{x})}{\eta \tilde{\eta}} \\
& =\frac{1}{\eta \tilde{\eta}}\left(-(|\eta-\tilde{\eta}|-\mathrm{i} \varepsilon)^{2}+\|\vec{x}-\overrightarrow{\tilde{x}}\|^{2}\right) \\
y_{+-}(x, \tilde{x}) & =\frac{1}{\eta \tilde{\eta}}\left(-(\eta-\tilde{\eta}+\mathrm{i} \varepsilon)^{2}+\|\vec{x}-\overrightarrow{\tilde{x}}\|^{2}\right)
\end{aligned}
$$




$$
\begin{aligned}
& y_{-+}(x, \tilde{x})=\frac{1}{\eta \tilde{\eta}}\left(-(\eta-\tilde{\eta}-\mathrm{i} \varepsilon)^{2}+\|\vec{x}-\overrightarrow{\tilde{x}}\|^{2}\right) \\
& y_{--}(x, \tilde{x})=\frac{1}{\eta \tilde{\eta}}\left(-(|\eta-\tilde{\eta}|+\mathrm{i} \varepsilon)^{2}+\|\vec{x}-\overrightarrow{\tilde{x}}\|^{2}\right),
\end{aligned}
$$

which all vanish near the lightcone. Here, $\varepsilon>0$ in (22) refers to the Feynman or time-ordered pole prescription $^{1}$. In de Sitter spacetime, i.e. when $\epsilon=0$, the de Sitter invariant function $y_{++}(x, \tilde{x})$ is related to the geodesic length $l(x, \tilde{x})$ as $\left.y_{++}\right|_{\varepsilon=0}=4 \sin ^{2}(H l / 2)$.

\section{FLRW fermion propagator: the massless case}

Massless fermions in FLRW spacetimes are not difficult to deal with. By making use of equation (9) the propagator for massless (conformal) fermions should satisfy

$$
a^{-\frac{D+1}{2}} \mathrm{i} \gamma^{b} \partial_{b}\left(a^{\frac{D-1}{2}} \mathrm{i} S_{c}(x, \tilde{x})\right)=\frac{\mathrm{i}}{a^{D}} \delta^{D}(x-\tilde{x}) .
$$

Let us recall the following identity:

$$
\partial^{2} \frac{1}{\Delta x_{++}^{D-2}(x, \tilde{x})}=\frac{4 \pi^{D / 2}}{\Gamma(D / 2-1)} \mathrm{i} \delta^{D}(x-\tilde{x}) .
$$

We can thus immediately infer the solution of equation (23):

$$
\mathrm{i} S_{c}(x, \tilde{x})=(a \tilde{a})^{-\frac{D-1}{2}} \frac{\Gamma(D / 2-1)}{4 \pi^{D / 2}} \mathrm{i} \gamma^{b} \partial_{b} \frac{1}{\Delta x_{++}^{D-2}(x, \tilde{x})} .
$$

Here, $\tilde{a}=a(\tilde{\eta})$. This completes the calculation for the propagator of massless fermions in FLRW spacetimes in $D$ dimensions. This propagator is valid in any FLRW spacetime and assumption ( $1 a$ ) can be relaxed. Because massless fermions are conformal in any dimension, their propagator is much easier to calculate than the massless scalar propagator [11-14].

\section{FLRW fermion propagator: the massive case}

A massive fermion is not conformal, and hence its propagator, in general, contains some complicated mass dependence ${ }^{2}$. We firstly solve for the fermionic mode functions. We generalize the approach outlined in $[40,41]$ to incorporate FLRW spacetimes with constant $\epsilon$. Using these mode functions, we then return to position space to construct the Feynman propagator.

\subsection{Fermionic mode functions}

Let us firstly define the rescaled fermionic spinor

$$
\chi(x)=a^{\frac{D-1}{2}}(\eta) \psi(x) .
$$

Keeping an eye on equation (9) one can easily check that this factor is chosen such to conveniently transform the covariant derivative into a partial derivative. Equation of motion (4) thus reads

$$
\mathrm{i} \gamma^{b} \partial_{b} \chi(x)-a m \chi(x)=0 .
$$

1 Note that the $\varepsilon$ of the Feynman pole prescription is unrelated to the 'slow-roll' parameter $\epsilon$ in FLRW spacetimes.

2 Note that in [36] there is an erroneous statement regarding massive fermion propagators in curved spacetimes. It is argued on page 87 that a fermionic propagator in any spacetime can be related to the scalar field propagator in that spacetime. This is not correct. The (spinorial) structure that arises when a covariant derivative acts on a spinor (9) is much more complicated than for scalar fields. 
The canonical momentum associated with $\hat{\psi}(x)$ follows from promoting the variation of (2) with respect to $\dot{\psi}(x)$ to an operator. According to the usual moves in quantum field theory, we impose anti-commutation relations between $\hat{\psi}(x)$ and its associated canonical momentum:

$$
\left\{\hat{\psi}_{a}(\mathbf{x}, t), a^{D-1}(t) \hat{\psi}_{b}^{*}(\mathbf{y}, t)\right\}=\delta^{D-1}(\mathbf{x}-\mathbf{y}) \delta_{a b},
$$

with the other anti-commutators vanishing. By making use of the rescaling in equation (26), appreciate that the anti-commutation relations above simplify:

$$
\begin{aligned}
& \left\{\hat{\chi}_{a}(\mathbf{x}, t), \hat{\chi}_{b}^{*}(\mathbf{y}, t)\right\}=\delta^{D-1}(\mathbf{x}-\mathbf{y}) \delta_{a b} \\
& \left\{\hat{\chi}_{a}(\mathbf{x}, t), \hat{\chi}_{b}(\mathbf{y}, t)\right\}=0 \\
& \left\{\hat{\chi}_{a}^{*}(\mathbf{x}, t), \hat{\chi}_{b}^{*}(\mathbf{y}, t)\right\}=0
\end{aligned}
$$

4.1.1. Chirality and helicity decomposition. Therefore, we expand the rescaled spinors $\hat{\chi}(x)$ and $\hat{\bar{\chi}}(x)$ in creation and annihilation operators as follows:

$$
\begin{aligned}
& \hat{\chi}(x)=\int \frac{\mathrm{d}^{D-1} \mathbf{k}}{(2 \pi)^{D-1}} \sum_{h} \hat{a}_{\mathbf{k}, h} \chi^{(h)}(\mathbf{k}, \eta) \mathrm{e}^{\mathrm{i} \mathbf{k} \cdot \mathbf{x}}+\hat{b}_{\mathbf{k}, h}^{\dagger} \nu^{(h)}(\mathbf{k}, \eta) \mathrm{e}^{-\mathrm{i} \mathbf{k} \cdot \mathbf{x}} \\
& \hat{\bar{\chi}}(x)=\int \frac{\mathrm{d}^{D-1} \mathbf{k}}{(2 \pi)^{D-1}} \sum_{h} \hat{b}_{\mathbf{k}, h} \bar{\nu}^{(h)}(\mathbf{k}, \eta) \mathrm{e}^{\mathrm{i} \mathbf{k} \cdot \mathbf{x}}+\hat{a}_{\mathbf{k}, h}^{\dagger} \bar{\chi}^{(h)}(\mathbf{k}, \eta) \mathrm{e}^{-\mathbf{i k} \cdot \mathbf{x}} .
\end{aligned}
$$

The expansion above merits a few remarks. Firstly, $\hat{a}_{\mathbf{k}, h}$ and $\hat{b}_{\mathbf{k}, h}$ are the fermion and antifermion annihilation operators of helicity $h$, respectively, in the usual sense: $\hat{a}_{\mathbf{k}, h}|\Omega\rangle=0=$ $\hat{b}_{\mathbf{k}, h}|\Omega\rangle$. The helicity $h$, i.e. the spin in the direction of motion, can be either +1 or -1 in units of $\hbar$.

Let us, for simplicity, return to the $D=4$ setting we are familiar with. We will generalize the following considerations shortly to arbitrary dimensions.

In the equation above $\chi^{(h)}(\mathbf{k}, \eta)$ is a 4 -spinor of helicity $h$. We decompose the 4-spinor $\chi(\mathbf{k}, \eta)$ into a direct product of chirality and helicity 2 -spinors:

$$
\chi(\mathbf{k}, \eta)=\sum_{h} \chi^{(h)}(\mathbf{k}, \eta)=\sum_{h}\left(\begin{array}{l}
\chi_{\mathrm{L}, h}(\mathbf{k}, \eta) \\
\chi_{\mathrm{R}, h}(\mathbf{k}, \eta)
\end{array}\right) \otimes \xi_{h} .
$$

Here, $\chi_{\mathrm{L}, h}(\mathbf{k}, \eta)$ and $\chi_{\mathrm{R}, h}(\mathbf{k}, \eta)$ are left- and right-handed 1-spinors of helicity $h$, respectively. Furthermore, $\xi_{h}$ is the helicity 2-eigenspinor:

$$
\hat{h} \xi_{h} \equiv \hat{k} \cdot \vec{\sigma} \xi_{h}=h \xi_{h},
$$

where $\vec{\sigma}=\left(\sigma^{1}, \sigma^{2}, \sigma^{3}\right)$ is a shorthand notation for the Pauli matrices. Furthermore, note that $\hat{h}^{2}=\hat{h}^{\dagger} \hat{h}=1$.

For future convenience, let us explicitly derive the two vectors $\xi_{h}$. We write

$$
\hat{\vec{k}}=\left(\hat{k}_{x}, \hat{k}_{y}, \hat{k}_{z}\right) \text {, }
$$

and keep in mind that

$$
\|\hat{\vec{k}}\|^{2}=\hat{k}_{x}^{2}+\hat{k}_{y}^{2}+\hat{k}_{z}^{2}=1 .
$$

It is a trivial exercise to solve for the two eigenvectors of $\hat{h}$, which correctly normalized to unity read 


$$
\begin{aligned}
& \xi_{+}=\frac{1}{\sqrt{2\left(1-\hat{k}_{z}\right)}}\left(\begin{array}{c}
\hat{k}_{x}-\mathrm{i} \hat{k}_{y} \\
1-\hat{k}_{z}
\end{array}\right) \\
& \xi_{-}=\frac{1}{\sqrt{2\left(1+\hat{k}_{z}\right)}}\left(\begin{array}{c}
\mathrm{i} \hat{k}_{y}-\hat{k}_{x} \\
1+\hat{k}_{z}
\end{array}\right) .
\end{aligned}
$$

Moreover, the helicity eigenstates are mutually orthogonal:

$$
\xi_{+}^{\dagger} \cdot \xi_{-}=0
$$

Finally, we have tacitly ignored the expansion of the anti-particle contribution $v(\mathbf{k}, \eta)$ in chirality and helicity spinors in equation (30). We will return to this subtlety shortly.

4.1.2. Generalization to higher dimensions. Our chirality and helicity decomposition of the spinor degrees of freedom in equation (31) also works in $D$ spacetime dimensions. Once a particular representation of the gamma matrices has been found, the projection operator,

$$
P_{ \pm}=\frac{1 \pm \gamma^{D+1}}{2}
$$

splits the $2^{D / 2}$-spinor degrees of freedom into two equal contributions of definite chirality, i.e., a left- and right-handed $2^{(D-2) / 2}$-spinor (see, e.g., [42]). Here

$$
\gamma^{D+1}=\alpha_{D} \gamma^{0} \cdots \gamma^{D-1},
$$

where $\alpha_{D}$ is fixed by requiring that $P_{ \pm}$is a proper projection operator:

$$
P_{ \pm}^{2}=P_{ \pm}, \quad P_{+} P_{-}=0 \quad \Longrightarrow \quad\left(\gamma^{D+1}\right)^{2}=1 .
$$

This yields

$$
\alpha_{D}=\exp \left[\mathrm{i} \frac{\pi}{4}(D-1)(D+2)\right] .
$$

In $D=4$, we recognize the familiar $\gamma^{5}=\mathrm{i} \gamma^{0} \gamma^{1} \gamma^{2} \gamma^{3}$. An helicity operator that commutes with the projection operator can be defined in Fourier space as

$$
\hat{h}=-\frac{\mathrm{e}^{\mathrm{i} \pi \frac{(D-2)(D-1)}{4}}}{\Gamma(D-1)} \epsilon^{i l_{1} \cdots l_{D-2}} k_{i} \gamma^{l_{1}} \cdots \gamma^{l_{D-2}},
$$

where $\epsilon^{i l_{1} \cdots l_{D-2}}$ is the Lévi-Civitá tensor in $D-1$ dimensions. The exponential phase ensures that $\hat{h}$ is Hermitian. Finally, the gamma function has been inserted to account for the number of different permutations of gamma matrices.

Hence, $\chi_{\mathrm{L}, h}(\mathbf{k}, \eta)$ and $\chi_{\mathrm{R}, h}(\mathbf{k}, \eta)$ are $2^{(D-4) / 2}$-spinors. Note that the definition of the helicity operator is consistent with (32). Most importantly, the chirality and helicity decomposition as proposed in equation (31) carries through for any dimension.

When an explicit form of $\xi_{h}$ is required, we return to $D=4$ and use equation (35). We subsequently generalize the result of such calculations by analytically extending it to arbitrary $D$.

4.1.3. Spinorial normalization conditions. In textbooks on quantum field theory, such as $[43,44]$, one expands the fermion in momentum space in spin eigenstates, whereas we expand in helicity eigenstates. This results in a different normalization requirement on the spinors in momentum space and consequently the standard textbook results cannot be straightforwardly copied. The reason for expanding in helicity eigenstates is that in curved spacetimes helicity is more convenient to work with rather than spin. 
Spin is a conserved quantity, i.e. an appropriate quantum number, in static backgrounds, whereas helicity is conserved in time dependent but spatially homogeneous backgrounds [45]. One can easily show that the helicity operator constructed in (41) commutes with the kinetic operator in Wigner space for spatially homogeneous correlators.

We normalize the spinors using two conditions: a consistent canonical quantization and orthogonality of particle and anti-particle spinors.

We thus impose the usual anti-commutation relations between creation and annihilation operators:

$$
\begin{aligned}
& \left\{\hat{a}_{\mathbf{k}, h}, \hat{a}_{\mathbf{k}^{\prime}, h^{\prime}}^{\dagger}\right\}=(2 \pi)^{D-1} \delta^{D-1}\left(\mathbf{k}-\mathbf{k}^{\prime}\right) \delta_{h, h^{\prime}} \\
& \left\{\hat{b}_{\mathbf{k}, h}, \hat{b}_{\mathbf{k}^{\prime}, h^{\prime}}^{\dagger}\right\}=(2 \pi)^{D-1} \delta^{D-1}\left(\mathbf{k}-\mathbf{k}^{\prime}\right) \delta_{h, h^{\prime}}
\end{aligned}
$$

and all other anti-commutators vanish identically. Now (42) is consistent with (29) if and only if

$$
\sum_{h} \chi_{a}^{(h)}(\mathbf{k}, \eta) \chi_{b}^{*(h)}(\mathbf{k}, \eta)+v_{a}^{(h)}(-\mathbf{k}, \eta) v_{b}^{*(h)}(-\mathbf{k}, \eta)=\delta_{a b}
$$

Note that this is a condition on $2^{(D / 2)-1}\left(2^{D / 2}+1\right)$ matrix elements in spinor space, i.e. for each $a$ and $b$ the above equality has to be satisfied.

Secondly, we require that the particle and anti-particle spinors are mutually orthogonal:

$$
\bar{\chi}^{(h)}(\mathbf{k}, \eta) v^{\left(h^{\prime}\right)}(\mathbf{k}, \eta)=0=\bar{v}^{\left(h^{\prime}\right)}(\mathbf{k}, \eta) \chi^{(h)}(\mathbf{k}, \eta) .
$$

This restricts four matrix elements in helicity space, i.e. for each $h, h^{\prime}= \pm$ the above equality needs to be obeyed.

Peskin and Schroeder [43] impose normalization conditions on both particle and antiparticle spinors in spin space. The corresponding conditions in helicity space are only partially satisfied. Both particle and anti-particle spinors of different helicity are trivially orthogonal by construction (36). However, we do not relate the amplitude of equal helicity states for particles and anti-particles to the mass of the spinor because helicity eigenstates for a particle at rest cannot be constructed.

4.1.4. Particle mode functions. We will now solve the Dirac equation. Let us insert equation (30) into the Dirac equation (27). Making use of our chirality and helicity decomposition (31) transforms the Dirac equation to a first-order coupled system of differential equations:

$$
\begin{aligned}
& \mathrm{i} \chi_{\mathrm{L}, h}^{\prime}+h k \chi_{\mathrm{L}, h}-a m \chi_{\mathrm{R}, h}=0 \\
& \mathrm{i} \chi_{\mathrm{R}, h}^{\prime}-h k \chi_{\mathrm{R}, h}-a m \chi_{\mathrm{L}, h}=0
\end{aligned}
$$

The arguments of the functions in the equation above have been omitted for notational convenience. The left- and right-handed spinors, however, are now a function of the magnitude of the Fourier mode $k$ only, because the differential equation is invariant under $\vec{k} \rightarrow-\vec{k}$.

Standard model fermions are chiral, in the sense that $m_{\mathrm{R}} \neq m_{\mathrm{L}}$. For simplicity, we take one mass $m=m_{\mathrm{R}}=m_{\mathrm{L}}$ only. The following derivation can easily be generalized when standard model fermions are considered by making use of the $P_{ \pm}$projectors in equation (37).

We now return to the expansion in chirality and helicity spinors of the anti-particle contribution $v(\mathbf{k}, \eta)$, which we did not need so far. Since the Dirac equation is a linear differential equation, the solution for $v(\mathbf{k}, \eta)$ cannot contain any new degrees of freedom once 
we completely solved for $\chi(\mathbf{k}, \eta)$. In other words, the form of $v(\mathbf{k}, \eta)$ is dictated once we have completely specified $\chi(\mathbf{k}, \eta)$. We expand $\nu(\mathbf{k}, \eta)$ slightly differently:

$$
v(\mathbf{k}, \eta)=\sum_{h} v^{(h)}(\mathbf{k}, \eta)=\sum_{h}\left(\begin{array}{l}
v_{\mathrm{R}, h}(\mathbf{k}, \eta) \\
v_{\mathrm{L}, h}(\mathbf{k}, \eta)
\end{array}\right) \otimes \xi_{h} .
$$

Note we flipped the position of the left- and right-handed spinors compared to (31). Consequently, the resulting equations of motion for both $v_{\mathrm{R}, h}(\mathbf{k}, \eta)$ and $v_{\mathrm{L}, h}(\mathbf{k}, \eta)$ are identical to $(45)$.

Alternatively, we could have expanded differently and flipped helicity, i.e. send $h \rightarrow-h$ in equation (31). Because the mass enforces mixing between different chirality states, labelling these states differently has no physical relevance.

We return to equation of motion (45) and define

$$
u_{ \pm h}(k, \eta) \equiv \frac{\chi_{\mathrm{L}, h}(k, \eta) \pm \chi_{\mathrm{R}, h}(k, \eta)}{\sqrt{2}} .
$$

This transforms equation (45) to

$$
\begin{aligned}
& \mathrm{i} u_{+h}^{\prime}+h k u_{-h}-a m u_{+h}=0 \\
& \mathrm{i} u_{-h}^{\prime}+h k u_{+h}+a m u_{-h}=0 .
\end{aligned}
$$

We exploit assumption (1a), which is equivalent to (21). Inserting this expression into the equation above yields

$$
\begin{aligned}
& \mathrm{i} u_{+h}^{\prime}+h k u_{-h}+\frac{m}{H(1-\epsilon)} \frac{u_{+h}}{\eta}=0 \\
& \mathrm{i} u_{-h}^{\prime}+h k u_{+h}-\frac{m}{H(1-\epsilon)} \frac{u_{-h}}{\eta}=0 .
\end{aligned}
$$

Recall that the mass of a fermion in Yukawa theory is time dependent in FLRW spacetimes. There does not exist a rescaling of the functions $u_{ \pm h}$ that removes the mass dependence in the equation of motion above. Even when one assumes that the mass $m$ is time independent, one cannot find such a rescaling. It is particularly simple to choose $m / H$ constant as the following from equation (16), and for convenience we define

$$
\zeta=\frac{m}{H(1-\epsilon)} .
$$

We can proceed and decouple the two linear differential equations to find

$$
u_{ \pm h}^{\prime \prime}+\left(k^{2}+\frac{\zeta^{2} \pm \mathrm{i} \zeta}{\eta^{2}}\right) u_{ \pm h}=0
$$

The solutions are given by

$$
u_{ \pm h}=\alpha_{ \pm k}^{h} \sqrt{-k \eta} H_{v_{ \pm}}^{(1)}(-k \eta)
$$

where $\alpha_{ \pm k}^{h}$ are two normalization constants that still need to be determined, and $H_{v_{ \pm}}^{(1)}$ is the Hankel function of the first kind of order:

$$
v_{ \pm}=\frac{1}{2} \mp \mathrm{i} \zeta
$$

This implies

$$
v_{+}+v_{-}=1
$$

10 


$$
v_{+}^{*}=v_{-} .
$$

The Hankel function of the second kind also solves equation (51). In the infinite asymptotic past, equivalent to the deep UV, the fermions become effectively massless for $\epsilon<1$, and the distinction between $u_{+h}$ and $u_{-h}$ vanishes as a consequence. Therefore, we require that the vacuum mode functions in this regime equal the standard conformal vacuum solutions:

$$
\begin{aligned}
& \lim _{\eta \rightarrow-\infty} u_{+h}(-k \eta)=\frac{1}{\sqrt{2}} \mathrm{e}^{-\mathrm{i} k \eta} \\
& \lim _{\eta \rightarrow-\infty} u_{-h}(-k \eta)=\frac{-h}{\sqrt{2}} \mathrm{e}^{-\mathrm{i} k \eta} .
\end{aligned}
$$

This excludes the Hankel function of the second kind for the moment from contributing. Away from the vacuum, we should, of course, also incorporate the second solution into (51) to allow for mode mixing. Note that the UV limits above yield the familiar UV behaviour for the leftand right-handed spinors (e.g., [43]):

$$
\begin{aligned}
& \lim _{\eta \rightarrow-\infty} \chi_{\mathrm{L}, h}(k, \eta)=\frac{1-h}{2} \mathrm{e}^{-\mathrm{i} k \eta} \\
& \lim _{\eta \rightarrow-\infty} \chi_{\mathrm{R}, h}(k, \eta)=\frac{1+h}{2} \mathrm{e}^{-\mathrm{i} k \eta} .
\end{aligned}
$$

When solutions (52) are substituted in (49a) one finds

$$
\alpha_{-k}^{h}=\mathrm{i} h \alpha_{+k}^{h} \mathrm{e}^{\mathrm{i} \pi v_{-}} .
$$

We are free to fix the remaining coefficient $\alpha_{+k}^{h}$ by the following condition:

$$
\sum_{ \pm}\left|u_{ \pm h}\right|^{2}=\left|u_{+h}\right|^{2}+\left|u_{-h}\right|^{2}=1
$$

We postpone the motivation for this requirement to section 4.1.6. For the moment, let us simply normalize accordingly for convenience. We thus find

$$
\alpha_{+k}^{h}=\sqrt{\frac{\pi}{4}} \mathrm{e}^{\mathrm{i} \frac{\pi}{2}\left(v_{+}+1 / 2\right)},
$$

where we have chosen the unobservable phase factor in accordance with the UV limit (55). We use some well-known identities involving Hankel functions which we list, for convenience, in appendix A.

Concluding, we find that the vacuum solutions to equation (49) neglecting derivatives of $\epsilon$ and treating the quotient $m / H$ as time independent are given by

$$
\begin{aligned}
& u_{+h}(-k \eta)=\mathrm{e}^{\mathrm{i} \frac{\pi}{2}\left(v_{+}+1 / 2\right)} \sqrt{-\frac{\pi k \eta}{4}} H_{v_{+}}^{(1)}(-k \eta) \\
& u_{-h}(-k \eta)=-h \mathrm{e}^{\mathrm{i} \frac{\pi}{2}\left(\nu_{-}+1 / 2\right)} \sqrt{-\frac{\pi k \eta}{4}} H_{\nu_{-}}^{(1)}(-k \eta) .
\end{aligned}
$$

As we send $\epsilon \rightarrow 0$ and approach de Sitter space, our solution is in accordance with [41], as can be seen from (53). In this solution, we have tacitly assumed that $\epsilon<1$ such that $\eta<0$, equivalent to an accelerated expansion. If however $\epsilon>1$ or $\eta>0$, which corresponds to a decelerating universe, one should simply use relations (A.1d)-(A.1e) in appendix A. The case $\epsilon=1$ requires special attention; see, e.g., [14] for the analogous scalar propagator case. 
Let us now turn our attention to finding the solution for the second mode function corresponding to the excited state. The procedure is completely analogous: we again let $\eta \rightarrow-\infty$ and match to the appropriate UV expansion involving the second solution $H_{v_{ \pm}}^{(2)}(-k \eta)$. We normalize the solution according to (58) and choose the phase factor in accordance with the corresponding UV limit. We can thus construct the total solution by linear superposition:

$$
\begin{aligned}
& u_{+h}(-k \eta)=c \mathcal{H}(\eta, \zeta)+\mathrm{d} \tilde{\mathcal{H}}(\eta, \zeta) \\
& u_{-h}(-k \eta)=-h c \tilde{\mathcal{H}}^{*}(\eta, \zeta)+h \mathrm{~d} \mathcal{H}^{*}(\eta, \zeta),
\end{aligned}
$$

where

$$
\begin{aligned}
& \mathcal{H}(\eta, \zeta)=\mathrm{e}^{\mathrm{i} \frac{\pi}{2}\left(v_{+}+1 / 2\right)} \sqrt{-\frac{\pi k \eta}{4}} H_{v_{+}}^{(1)}(-k \eta) \\
& \tilde{\mathcal{H}}(\eta, \zeta)=\mathrm{e}^{-\mathrm{i} \frac{\pi}{2}\left(v_{+}+1 / 2\right)} \sqrt{-\frac{\pi k \eta}{4}} H_{v_{+}}^{(2)}(-k \eta) .
\end{aligned}
$$

Note that we have omitted all $k$ dependence in (61) for notational convenience. Note finally that the two fundamental solutions (62) in $u_{+h}(-k \eta)$ are not complex conjugates of each other, unlike, for example, for the scalar field case. However, the second solution $u_{-h}(-k \eta)$ can be obtained from complex conjugation of the fundamental solutions comprising $u_{+h}(-k \eta)$, as can clearly be seen in equation (61).

For the moment, we leave the normalization constants $c$ and $d$ undetermined. Appreciate, we are nevertheless free to normalize the fundamental solutions to unity. Of course, we will determine the normalization constants shortly by applying our analysis performed in subsection 4.1.3. But first, we need to solve for the anti-particle mode functions.

4.1.5. Anti-particle mode functions. Let us stress again that the other spinor $v^{(h)}(\mathbf{k}, \eta)$ cannot contain any new degrees of freedom because Dirac's equation is linear. We only have to determine the connection with $\chi^{(h)}(\mathbf{k}, \eta)$. When we expand according to (46) the coupled linear differential equations $\nu_{\mathrm{R}, h}(\mathbf{k}, \eta)$ and $v_{\mathrm{L}, h}(\mathbf{k}, \eta)$ obey are identical to (45). Therefore, we can analogously define

$$
\nu_{ \pm h}(k, \eta) \equiv \frac{\nu_{\mathrm{L}, h}(k, \eta) \pm v_{\mathrm{R}, h}(k, \eta)}{\sqrt{2}} .
$$

We are rewarded for our extensive discussion of chirality and helicity. The solutions of $v_{ \pm h}(-k \eta)$ are identical to (61), where we should only denote the coefficients in this solution differently:

$$
\begin{aligned}
& v_{+h}(-k \eta)=f \mathcal{H}(\eta, \zeta)+g \tilde{\mathcal{H}}(\eta, \zeta) \\
& v_{-h}(-k \eta)=-h f \tilde{\mathcal{H}}^{*}(\eta, \zeta)+h g \mathcal{H}^{*}(\eta, \zeta)
\end{aligned}
$$

The functions appearing in this equation are given by (62). We need to determine how the coefficients $c$ and $d$ of $u_{ \pm h}(-k \eta)$ relate to $f$ and $g$ of $v_{ \pm h}(-k \eta)$. Moreover, we need to find the correct normalization condition for $c$ and $d$. 
4.1.6. Normalization of the mode functions. Inspired by our discussion in 4.1.3, we impose two normalization conditions on the spinors: a consistent canonical quantization (43) and orthogonality between particle and anti-particle spinors (44).

After quite some work the, naively, ten constraint equations in $D=4$ in (43) yield the following three conditions on our coefficients:

$$
\begin{aligned}
& |c|^{2}+|d|^{2}+|f|^{2}+|g|^{2}=2 \\
& |c|^{2}-|d|^{2}+|f|^{2}-|g|^{2}=0 \\
& c d^{*}+f g^{*}=0 .
\end{aligned}
$$

The orthogonality condition (44) yields one more condition:

$$
c f^{*}+d g^{*}=0
$$

The solution is

$$
\begin{aligned}
& |f|=|d| \\
& |g|=|c| \\
& \phi_{f}=\phi_{c}-\phi_{d}+\phi_{g} \pm \pi .
\end{aligned}
$$

The last line relates the phases of our normalization constants, e.g., $\phi_{f}$ denotes the phase of $f$. Moreover, from (65) we can derive

$$
\begin{aligned}
& |c|^{2}+|d|^{2}=1 \\
& |f|^{2}+|g|^{2}=1,
\end{aligned}
$$

where the second line is a consequence of the first. Note that this condition also follows from charge conservation as argued in [41]. Changing the normalization in (58) results in a change of the right-hand side of equations $(65 a),(66 d)$ and $(66 e)$. Normalizing the fundamental solutions to unity as in (58) is particularly convenient as it allows for a particle interpretation of $|d|^{2}$ and $|f|^{2}$.

The normalization constants provide us with three physical degrees of freedom. Of course, $|c|$ is physical which determines $|d|$ through (66d). Hence, $\phi_{d}$ is physical just as in the scalar field case. Since $\phi_{c}$ is a phase that can be removed without physical consequences, the phase relation (66c) determines $\phi_{f}$ in terms of the third physical phase $\phi_{g}$. Note finally that one of the two phase relations $(65 c)$ and $(65 d)$ is redundant in this part of the analysis.

A final remark is in order. To derive (65), we needed the explicit form of $\xi_{h}$ in equation (35), which is only valid in $D=4$. However, we analytically continue this result to arbitrary dimension and one naturally finds (65).

4.1.7. Summary. Using the solutions for $u_{ \pm h}(k, \eta)$ and $v_{ \pm h}(k, \eta)$ given in (61) and (64) respectively, we recall relations (47) and (63) to find

$$
\begin{aligned}
& \chi_{\mathrm{L}, h}(k, \eta)=\frac{1}{\sqrt{2}}\left[c\left\{\mathcal{H}-h \tilde{\mathcal{H}}^{*}\right\}+d\left\{\tilde{\mathcal{H}}+h \mathcal{H}^{*}\right\}\right] \\
& \chi_{\mathrm{R}, h}(k, \eta)=\frac{1}{\sqrt{2}}\left[c\left\{\mathcal{H}+h \tilde{\mathcal{H}}^{*}\right\}+d\left\{\tilde{\mathcal{H}}-h \mathcal{H}^{*}\right\}\right],
\end{aligned}
$$

and moreover, 


$$
\begin{aligned}
& v_{\mathrm{L}, h}(k, \eta)=\frac{1}{\sqrt{2}}\left[f\left\{\mathcal{H}-h \tilde{\mathcal{H}}^{*}\right\}+g\left\{\tilde{\mathcal{H}}+h \mathcal{H}^{*}\right\}\right] \\
& v_{\mathrm{R}, h}(k, \eta)=\frac{1}{\sqrt{2}}\left[f\left\{\mathcal{H}+h \tilde{\mathcal{H}}^{*}\right\}+g\left\{\tilde{\mathcal{H}}-h \mathcal{H}^{*}\right\}\right],
\end{aligned}
$$

where the fundamental solutions are given in equation (62). The normalization conditions (66) determine $f$ and $g$ in terms of $c$ and $d$. Moreover, we have $|c|^{2}+|d|^{2}=1$.

\subsection{The Feynman propagator}

Having discussed the fermionic mode functions in some detail, we can turn our attention to solving the Feynman propagator for fermions. We insert the rescaled spinors, expanded in terms of creation and annihilation operators, into the formal definition of the Feynman propagator (19), which yields

$$
\begin{aligned}
\mathrm{i} S_{\mathrm{F}}^{a b}(x, \tilde{x})=\theta & (\eta-\tilde{\eta}) a^{-\frac{D-1}{2}} \tilde{a}^{-\frac{D-1}{2}} \int \frac{\mathrm{d}^{D-1} \mathbf{k}}{(2 \pi)^{D-1}} \sum_{h} \chi_{a}^{(h)}(\mathbf{k}, \eta) \bar{\chi}_{b}^{(h)}(\mathbf{k}, \tilde{\eta}) \mathrm{e}^{\mathrm{i} \mathbf{k} \cdot(\mathbf{x}-\tilde{\mathbf{x}})} \\
& -\theta(\tilde{\eta}-\eta) a^{-\frac{D-1}{2}} \tilde{a}^{-\frac{D-1}{2}} \int \frac{\mathrm{d}^{D-1} \mathbf{k}}{(2 \pi)^{D-1}} \sum_{h} \bar{v}_{b}^{(h)}(\mathbf{k}, \tilde{\eta}) v_{a}^{(h)}(\mathbf{k}, \eta) \mathrm{e}^{-\mathrm{i} \mathbf{k} \cdot(\mathbf{x}-\tilde{\mathbf{x}})},
\end{aligned}
$$

where $a=a(\eta)$ and $\tilde{a}=a(\tilde{\eta})$. The reader can easily see that we have to make use of solutions (67) to calculate the 16 matrix elements of the propagator.

4.2.1. Outline of the calculation. Let us outline this rather cumbersome calculation in seven steps:

1. We recall the form of, e.g., the massless fermionic propagator (25) and realize that we have to extract an operator acting on $x$, rather than on $\tilde{x}$.

The reader can easily see that the 16 matrix elements of the propagator contain products of two Hankel functions. Some of these products involve Hankel functions at the same order, but others encompass Hankel functions where the order differs. Since we can only easily integrate products of Hankel functions of equal order we need to transform precisely the latter products by making use of identities (A.1), e.g.,

$H_{v_{+}}^{(m)}(-k \eta) H_{v_{-}}^{(n)}(-k \tilde{\eta})=-\frac{\mathrm{e}^{ \pm \mathrm{i} \pi\left(\nu_{-}-1\right)}}{k}\left(\frac{\mathrm{d}}{\mathrm{d} \eta}+\frac{\nu_{-}}{\eta}\right) H_{\nu_{-}}^{(m)}(-k \eta) H_{v_{-}}^{(n)}(-k \tilde{\eta})$,

where + or - applies when $m$ equals 1 or 2 , respectively. Clearly, the 'extracted operator' is a function of $\eta$.

2. We rewrite the Hankel functions in terms of MacDonald functions. By making use of $[46,47]$ we find

$$
\begin{aligned}
& H_{v}^{(1)}(z)=-\frac{2 \mathrm{i}}{\pi} \mathrm{e}^{-\mathrm{i} \frac{\pi}{2} v} K_{v}(-\mathrm{i} z) \\
& H_{v}^{(2)}(z)=\frac{2 \mathrm{i}}{\pi} \mathrm{e}^{\mathrm{i} \frac{\pi}{2} v} K_{v}(\mathrm{i} z)
\end{aligned}
$$

where $K_{v}(z)$ is a MacDonald function.

3 . The emerging matrix structure of equation (68) is most easily uncovered by evaluating its $2 \times 2$-matrix constituents successively and writing the result in terms of Pauli matrices. Recalling equations (33) and (34) we derive

$$
k\left(\hat{k}_{x} \sigma_{x}+\hat{k}_{y} \sigma_{y}+\hat{k}_{z} \sigma_{z}\right)=k_{j} \sigma^{j} \rightarrow \pm \mathrm{i} \sigma^{j} \partial_{j}
$$


where the - or + sign applies depending on whether one deals with the $\theta(\eta-\tilde{\eta})$ or $\theta(\tilde{\eta}-\eta)$ contribution in (68), respectively. The last step is possible because only the exponent depends on $\mathbf{x}$. Note finally that the index $j$ in equation (71) is supposed to run over (flat) spatial indices exclusively.

4. The $\mathbf{k}$ independent contribution to the propagator can thus be pulled out of the Fourier integral. These matrices simplify considerably when we consider the following identities:

$\left(\mathrm{i} \gamma^{b} \partial_{b}+a m\right) \frac{1 \pm \gamma^{0}}{2}=\frac{1}{2}\left(\begin{array}{cc} \pm \mathrm{i} \partial_{\eta} \pm \mathrm{i} \sigma^{j} \partial_{j}+a m & \mathrm{i} \partial_{\eta}+\mathrm{i} \sigma^{j} \partial_{j} \pm a m \\ \mathrm{i} \partial_{\eta}-\mathrm{i} \sigma^{j} \partial_{j} \pm a m & \pm \mathrm{i} \partial_{\eta} \mp \mathrm{i} \sigma^{j} \partial_{j}+a m\end{array}\right)$.

Clearly, we recognize the complex conjugate of the Dirac operator and the $\left(1 \pm \gamma^{0}\right) / 2$ structure we are familiar with from the de Sitter propagator.

5. Let us present an intermediate result that already captures much of the final structure of our propagator. For brevity, let us only consider the contribution to the propagator arising from the first $\theta$-function in the propagator, i.e. when $\eta>\tilde{\eta}$. This part of the Feynman propagator reads

$$
\begin{aligned}
\left.\mathrm{i} S_{\mathrm{F}}^{a b}(x, \tilde{x})\right|_{\eta>\tilde{\eta}} & =a^{-\frac{D-1}{2}} \tilde{a}^{-\frac{D-1}{2}}\left(\mathrm{i} \gamma^{b} \partial_{b}+a m\right)\left[\frac { \sqrt { \eta \tilde { \eta } } } { \pi } \left\{\frac{1+\gamma^{0}}{2} \int \frac{\mathrm{d}^{D-1} \mathbf{k}}{(2 \pi)^{D-1}} K_{-}(k, \eta, \tilde{\eta})\right.\right. \\
& \left.\left.+\frac{1-\gamma^{0}}{2} \int \frac{\mathrm{d}^{D-1} \mathbf{k}}{(2 \pi)^{D-1}} K_{+}(k, \eta, \tilde{\eta})\right\}\right]
\end{aligned}
$$

where

$$
\begin{aligned}
K_{-}(k, \eta, \tilde{\eta})= & |c|^{2} K_{\nu_{-}}(\mathrm{i} k \eta) K_{\nu_{-}}(-\mathrm{i} k \tilde{\eta})-\mathrm{i} c d^{*} K_{\nu_{-}}(\mathrm{i} k \eta) K_{\nu_{-}}(\mathrm{i} k \tilde{\eta}) \\
& -\mathrm{i} c^{*} d K_{\nu_{-}}(-\mathrm{i} k \eta) K_{\nu_{-}}(-\mathrm{i} k \tilde{\eta})-|d|^{2} K_{\nu_{-}}(-\mathrm{i} k \eta) K_{\nu_{-}}(\mathrm{i} k \tilde{\eta}),
\end{aligned}
$$

and where

$$
\begin{aligned}
K_{+}(k, \eta, \tilde{\eta})= & |c|^{2} K_{v_{+}}(\mathrm{i} k \eta) K_{v_{+}}(-\mathrm{i} k \tilde{\eta})+\mathrm{i} c d^{*} K_{v_{+}}(\mathrm{i} k \eta) K_{v_{+}}(\mathrm{i} k \tilde{\eta}) \\
& +\mathrm{i} c^{*} d K_{v_{+}}(-\mathrm{i} k \eta) K_{v_{+}}(-\mathrm{i} k \tilde{\eta})-|d|^{2} K_{v_{+}}(-\mathrm{i} k \eta) K_{v_{+}}(\mathrm{i} k \tilde{\eta}) .
\end{aligned}
$$

The contribution to the propagator when $\eta<\tilde{\eta}$ is given by the same expression, where we only need to replace the normalization constants $c$ and $d$ by $f$ and $g$, respectively.

The complicated structure of the propagator in spinor space has dramatically simplified by extracting the appropriate operators in position space, which could already be expected from the propagator in de Sitter spacetime.

6. We need to perform the Fourier integrals over the functions $K_{-}(k, \eta, \tilde{\eta})$ and $K_{+}(k, \eta, \tilde{\eta})$ next. We use

$$
\int \frac{\mathrm{d}^{D-1} \mathbf{k}}{(2 \pi)^{D-1}} \mathrm{e}^{\mathrm{i} \mathbf{k} \cdot \mathbf{x}} f(k)=\frac{2}{(4 \pi)^{\frac{D-1}{2}}} \int_{0}^{\infty} \mathrm{d} k k^{D-2} \frac{J_{\frac{D-3}{2}}(k x)}{\left(\frac{1}{2} k x\right)^{\frac{D-3}{2}}} f(k),
$$

which is valid for any function $f(k)$ that depends solely on $k=\|\mathbf{k}\|$. Here, $J_{\mu}(k x)$ is a Bessel function of the first kind. The reader can easily verify that all the Fourier integrals we need to perform are of the following form:

$$
\int_{0}^{\infty} \mathrm{d} k k^{\mu+1} J_{\mu}(c k) K_{v}(\alpha k) K_{v}(\beta k),
$$

where the arguments of the MacDonald functions are purely imaginary:

$$
\begin{aligned}
& \alpha= \pm \mathrm{i} \eta \\
& \beta= \pm \mathrm{i} \tilde{\eta}
\end{aligned}
$$


We perform these integrals in appendix B by making use of [46]. The result of this integral can be expressed in terms of the Gauss' hypergeometric function ${ }_{2} F_{1}$. The $\theta$-functions in the propagator in combination with the arguments of the hypergeometric functions allow us to rewrite these arguments in terms of the $y$-functions (22) we introduced in section 2.3.

A well-known fact in Minkowski or de Sitter spacetime extends to our analysis in FLRW spacetimes: the small real contribution needed to make the Fourier integrals convergent determines the $\varepsilon$ pole prescription of the various contributions to the propagator.

7. The final result for the constant $\epsilon$ and constant $\zeta$ FLRW fermion propagator reads

$\mathrm{i} S_{F}^{a b}(x, \tilde{x})=a\left(\mathrm{i} \gamma^{\mu} \nabla_{\mu}+m\right) \frac{(a \eta \tilde{a} \tilde{\eta})^{-\frac{D-2}{2}}}{\sqrt{a \tilde{a}}}\left[\mathrm{i} S_{+}(x, \tilde{x}) \frac{1+\gamma^{0}}{2}+\mathrm{i} S_{-}(x, \tilde{x}) \frac{1-\gamma^{0}}{2}\right]$,

where

$$
\begin{aligned}
& \mathrm{i} S_{ \pm}(x, \tilde{x})= \frac{\Gamma\left(\frac{D}{2} \pm \mathrm{i} \zeta\right) \Gamma\left(\frac{D-2}{2} \mp \mathrm{i} \zeta\right)}{(4 \pi)^{D / 2} \Gamma\left(\frac{D}{2}\right)}\left\{|c|^{2}{ }_{2} F_{1}\left(\frac{D}{2} \pm \mathrm{i} \zeta, \frac{D-2}{2} \mp \mathrm{i} \zeta ; \frac{D}{2} ; 1-\frac{y_{++}(x ; \tilde{x})}{4}\right)\right. \\
& \mp \mathrm{i} c d^{*} \mathrm{e}^{\mathrm{i} \pi \frac{D-1}{2}}{ }_{2} F_{1}\left(\frac{D}{2} \pm \mathrm{i} \zeta, \frac{D-2}{2} \mp \mathrm{i} \zeta ; \frac{D}{2} ; 1-\frac{y_{+-}(x ; \tilde{\bar{x}})}{4}\right) \\
& \mp \mathrm{i} c^{*} d \mathrm{e}^{-\mathrm{i} \pi \frac{D-1}{2}}{ }_{2} F_{1}\left(\frac{D}{2} \pm \mathrm{i} \zeta, \frac{D-2}{2} \mp \mathrm{i} \zeta ; \frac{D}{2} ; 1-\frac{y_{-+}(x ; \tilde{\bar{x}})}{4}\right) \\
&\left.-|d|^{2}{ }_{2} F_{1}\left(\frac{D}{2} \pm \mathrm{i} \zeta, \frac{D-2}{2} \mp \mathrm{i} \zeta ; \frac{D}{2} ; 1-\frac{y_{--}(x ; \tilde{x})}{4}\right)\right\},
\end{aligned}
$$

where the $y$-functions are given in equation (22) and $\zeta$ in (50). Moreover, to write the propagator in terms of the $y$-functions we introduced the following notation:

$$
\tilde{\bar{x}}=\overline{(\tilde{\eta}, \tilde{\mathbf{x}})}=(-\tilde{\eta}, \tilde{\mathbf{x}}) \text {. }
$$

One can think of $\tilde{\bar{x}}$ as an antipodal coordinate (see, e.g., [13, 28, 48-50]). Note that we extract an operator in (78) that does not depend on $\tilde{x}$. However, there is nothing special about the $x$ leg of the propagator. Keeping remark 1 above in mind, we know that we could have equally well written the propagator (78) in terms of an operator acting on $\tilde{x}$ by replacing

$$
a\left(\mathrm{i} \gamma^{\mu} \nabla_{\mu}+m\right) \rightarrow \tilde{a}\left(\mathrm{i} \tilde{\gamma}^{\mu} \tilde{\nabla}_{\mu}+m\right) .
$$

This propagator would then have satisfied the Dirac equation (20) in terms of $\tilde{x}$.

As a first check of this result, note that if we send $m \rightarrow 0$ and $d \rightarrow 0$, the massive fermionic FLRW propagator (78) correctly yields the massless fermionic FLRW propagator earlier derived in equation (25). Again, in order to derive this result we needed the explicit form of $\xi_{h}$ given by (35). The operator in (78) has thus been constructed in $D=4$. However, we can analytically continue again to arbitrary dimensions.

Finally, let us study the IR behaviour of our propagator. Note that the coincidence propagator (in Fourier space) is IR finite due to the Pauli exclusion principle. In position space, note that the IR limit of all the $y$-functions in equation (22) is

$$
y(x ; \tilde{x}) \rightarrow \pm \infty,
$$

where the plus or minus sign applies for infinite spacelike or timelike separation, respectively. If we recall the following well-known relation,

$$
\begin{aligned}
{ }_{2} F_{1}(a, b ; c ; z) & =\frac{\Gamma(c) \Gamma(b-a)}{\Gamma(b) \Gamma(c-a)}(1-z)^{-a}{ }_{2} F_{1}\left(a, c-b ; a-b+1 ; \frac{1}{1-z}\right) \\
& +\frac{\Gamma(c) \Gamma(a-b)}{\Gamma(a) \Gamma(c-b)}(1-z)^{-b}{ }_{2} F_{1}\left(b, c-a ; b-a+1 ; \frac{1}{1-z}\right),
\end{aligned}
$$


we can easily extract the leading-order IR behaviour of the propagator in equation (79):

$$
\begin{aligned}
\mathrm{i} S_{ \pm}(x, \tilde{x})= & \frac{\Gamma\left(\frac{D-2}{2} \mp \mathrm{i} \zeta\right) \Gamma(1 \pm 2 \mathrm{i} \zeta)}{(4 \pi)^{D / 2} \Gamma(1 \pm \mathrm{i} \zeta)}\left\{|c|^{2}\left(\frac{y_{++}(x ; \tilde{x})}{4}\right)^{-\left(\frac{D-2}{2} \pm \mathrm{i} \zeta\right)}\right. \\
& \mp \mathrm{i} c d^{*} \mathrm{e}^{\mathrm{i} \pi \frac{D-1}{2}}\left(\frac{y_{+-}(x ; \tilde{\bar{x}})}{4}\right)^{-\left(\frac{D-2}{2} \pm \mathrm{i} \zeta\right)} \mp \mathrm{i} c^{*} d \mathrm{e}^{-\mathrm{i} \pi \frac{D-1}{2}}\left(\frac{y_{-+}(x ; \tilde{\bar{x}})}{4}\right)^{-\left(\frac{D-2}{2} \pm \mathrm{i} \zeta\right)} \\
& \left.-|d|^{2}\left(\frac{y_{--}(x ; \tilde{x})}{4}\right)^{-\left(\frac{D-2}{2} \pm \mathrm{i} \zeta\right)}\right\}+\mathcal{O}\left(y^{\frac{D}{2} \pm \mathrm{i} \zeta}\right)
\end{aligned}
$$

Note that for the spacelike IR, all $y$-functions behave identically and the result above can be simplified further. For the timelike IR, the $\varepsilon$-prescription in equation (22) dictates how to take the powers of the $y$-functions. Of course, one has to insert equation (84) into (78) to obtain the full propagator in the IR limit.

The fermionic propagator (78) is analogous to the scalar and the graviton propagator in universes with the constant deceleration parameter $q=\epsilon-1$ derived in [12-14]. Unlike in the case of scalars and gravitons, where quite generically the Bunch-Davies vacuum is IR divergent, the fermionic propagator is IR finite due to the Pauli exclusion principle which forbids an accumulation of fermions in the deep IR. The IR behaviour for the scalar and graviton is important, as it can comprise secular effects (that grow as a power in time) in, for example, $V_{\text {eff }}(\Phi)$ (see [51]) and $T_{1 \text { loop }}^{\mu \nu}$ (see [14, 52]). Mathematically speaking, Pauli blocking is enforced by the imaginary $i$ in equation (51), which ultimately carries through in the complex indices of the hypergeometric functions in the propagator (79).

\subsection{The fermionic de Sitter propagator}

Having derived the fermionic FLRW propagator, let us verify whether it correctly reduces to the fermionic de Sitter propagator, which is a well-known result in the literature. The fermion propagator in de Sitter spacetime reads $[15,19]$

$\mathrm{i} S_{F}^{\Lambda}(x, \tilde{x})=a\left(\mathrm{i} \gamma^{\mu} \nabla_{\mu}+m\right) \frac{H^{D-2}}{\sqrt{a \tilde{a}}}\left[\mathrm{i} S_{+}^{\Lambda}(x, \tilde{x}) \frac{1+\gamma^{0}}{2}+\mathrm{i} S_{-}^{\Lambda}(x, \tilde{x}) \frac{1-\gamma^{0}}{2}\right]$,

where the superscript $\Lambda$ denotes de Sitter spacetime and where

$\mathrm{i} S_{ \pm}^{\Lambda}(x, \tilde{x})=\frac{1}{(4 \pi)^{D / 2}} \frac{\Gamma\left(\frac{D}{2}-1 \mp \mathrm{i} \frac{m}{H}\right) \Gamma\left(\frac{D}{2} \pm \mathrm{i} \frac{m}{H}\right)}{\Gamma\left(\frac{D}{2}\right)}{ }_{2} F_{1}\left(\frac{D}{2}-1 \mp \mathrm{i} \frac{m}{H}, \frac{D}{2} \pm \mathrm{i} \frac{m}{H} ; \frac{D}{2} ; 1-\frac{y}{4}\right)$.

Here, $y$ is shorthand for $y_{++}(x, \tilde{x})$. If we send $\epsilon \rightarrow 0$ and set $c=1$ and $d=0$, equation (78) correctly reproduces the de Sitter result. Moreover, using

$$
{ }_{2} F_{1}\left(\frac{D}{2}-1, \frac{D}{2} ; \frac{D}{2} ; 1-\frac{y}{4}\right)=\left(\frac{y}{4}\right)^{1-D / 2},
$$

note that the massless limit of (85) indeed corresponds to (25) in de Sitter space. We conclude that the fermionic propagator in FLRW spacetimes correctly reduces to the known cases in the existing literature.

\section{One-loop effective action}

As a simple application for the propagator derived in section 4.2, we calculate the one-loop contribution to the effective action. This is important to study the impact of fermions on the 
evolution of the background spacetime and of scalar fields coupled to these fermions. We renormalize using the minimal subtraction dimensional renormalization technique.

\subsection{Evaluating the one-loop backreaction}

The one-loop effective action formally reads

$$
\begin{aligned}
\Gamma_{1} & =-\mathrm{i} \operatorname{Tr} \log \left[\sqrt{-g}\left(\mathrm{i} \gamma^{\mu} \nabla_{\mu}-m\right)\right] \\
& =\int^{m} \mathrm{~d} \bar{m} \operatorname{Tr}\left[\sqrt{-g} \mathrm{i} S_{\mathrm{F}}^{a b}(x, \tilde{x})\right] .
\end{aligned}
$$

Here, the trace is both over spatial and spinorial indices. We have been able to evaluate the logarithm at the expense of losing all mass-independent contributions to the effective action.

The effective action for a massless fermion is completely fixed by the trace anomaly which this field is known to exhibit (see, for example, [25, 26, 36]). The trace anomaly can be generated from a finite non-local effective action (see, e.g., [25]). It can alternatively be generated from an infinite but local effective action [26]. We will henceforth neglect all contributions from the trace anomaly to the effective action in this paper, because it has already been extensively discussed in the literature.

We treat the covariant derivative according to (9), and we can easily expand the hypergeometric functions in equation (79) at coincidence. In dimensional regularization, the $D$-dependent powers in this expansion do not contribute. These are generated for the $y_{++}$ and $y_{--}$contributions to (79) by the first term in

$$
\begin{gathered}
{ }_{2} F_{1}(a, b ; c ; z)=\frac{\Gamma(c) \Gamma(a+b-c)}{\Gamma(a) \Gamma(b)}(1-z)^{c-a-b}{ }_{2} F_{1}(c-a, c-b ; c-a-b+1 ; 1-z) \\
+\frac{\Gamma(c) \Gamma(c-a-b)}{\Gamma(c-a) \Gamma(c-b)}{ }_{2} F_{1}(a, b ; a+b-c+1 ; 1-z) .
\end{gathered}
$$

Moreover, when we trace over spacetime indices note that there is no contribution at all from the derivative term hitting one of the hypergeometric functions:

$$
\lim _{\tilde{x} \rightarrow x} \sum_{n=0}^{\infty} \gamma^{b} \partial_{b} y_{++}^{n}(x, \tilde{x})=-\lim _{\tilde{x} \rightarrow x} \sum_{n=0}^{\infty} n \gamma^{0} \frac{y_{++}^{n}(x, \tilde{x})}{\eta}=0 .
$$

Here, $n$ is the integer-valued coefficient of the Taylor expansion of a hypergeometric function. A similar identity holds for $y_{--}$. Likewise, for $y_{+-}$or $y_{-+}$, we have, e.g.,

$$
\lim _{\tilde{x} \rightarrow x} \gamma^{b} \partial_{b} y_{+-}(x, \tilde{\bar{x}})=0 .
$$

Note that both $y_{+-}$and $y_{-+}$depend on the antipodal coordinate $\tilde{\bar{x}}$ in equation (78). At coincidence, we also use

$$
(a \tilde{a})^{-\frac{D-1}{2}}(\eta \tilde{\eta})^{-\frac{D-2}{2}}\left(a m \mp \frac{D-2}{2} \frac{\mathrm{i}}{\eta}\right) \rightarrow \pm \mathrm{i}(H|1-\epsilon|)^{D-2} H(1-\epsilon)\left(\frac{D-2}{2} \mp \mathrm{i} \zeta\right)
$$

The result is

$$
\begin{aligned}
\Gamma_{1}=\int \mathrm{d}^{D} x & \sqrt{-g} \frac{(H|1-\epsilon|)^{D-2}}{(2 \pi)^{D / 2}}\left\{\Gamma\left(1-\frac{D}{2}\right)\left(|c|^{2}-|d|^{2}\right) \int^{m} \mathrm{~d} \tilde{m} \tilde{m} \frac{\Gamma\left(\frac{D}{2}+\mathrm{i} \tilde{\zeta}\right) \Gamma\left(\frac{D}{2}-\mathrm{i} \tilde{\zeta}\right)}{\Gamma(1-\mathrm{i} \tilde{\zeta}) \Gamma(1+\mathrm{i} \tilde{\zeta})}\right. \\
& \left.+H(1-\epsilon)\left(c d^{*} \mathrm{e}^{\mathrm{i} \pi \frac{D-1}{2}}+c^{*} d \mathrm{e}^{-\mathrm{i} \pi \frac{D-1}{2}}\right) \int^{m} \mathrm{~d} \tilde{m} \frac{\Gamma\left(\frac{D}{2}+\mathrm{i} \tilde{\zeta}\right) \Gamma\left(\frac{D}{2}-\mathrm{i} \tilde{\zeta}\right)}{\Gamma\left(\frac{D}{2}\right)}\right\} . \quad \text { (93) }
\end{aligned}
$$


Let us compare this result with the known calculations in the literature in de Sitter spacetime $(\epsilon \rightarrow 0)$ and in the vacuum $(|d|=0)$. Note the two omissions in the Candelas and Raine effective action [15]. When tracing over spinor indices, we have

$$
\operatorname{Tr} \frac{1 \pm \gamma^{0}}{2}=2^{\frac{D}{2}-1}
$$

and this trace does not, as is apparent from [15], equal $D / 2$ because a spinor in $D$ spacetime dimensions has $2^{\frac{D}{2}}$ degrees of freedom (see, e.g., [42]). Indeed, this has already been noted by [53, 54]. Moreover, the effective action in [15] misses a factor of 2 stemming from the separate particle and anti-particle contributions, which has been corrected for in, e.g., [19].

The reader can easily verify that (93), taking these errors into account, agrees with $[15,19]$ in de Sitter spacetime and in the vacuum.

\subsection{Dimensional regularization}

In the spirit of dimensional regularization, we now expand around $D=4$. We can use the familiar result:

$$
\Gamma(x+y \tilde{\epsilon})=\Gamma(x)[1+y \tilde{\epsilon} \psi(x)]+\mathcal{O}\left(\tilde{\epsilon}^{2}\right),
$$

where $\tilde{\epsilon} \ll 1$ and where $\psi(x)$ is the digamma function defined by $\psi(x)=\mathrm{d} \log \Gamma(x) / \mathrm{d} x$. Anticipating the form of the counterterms we will add shortly, we introduce a scale $\mu$ by

$$
\begin{aligned}
(H|1-\epsilon|)^{D} & =(H(1-\epsilon))^{4} \mu^{D-4}\left(\frac{H|1-\epsilon|}{\mu}\right)^{D-4} \\
& \simeq(H(1-\epsilon))^{4} \mu^{D-4}\left[1+\frac{D-4}{2} \ln \left(\frac{H^{2}(1-\epsilon)^{2}}{\mu^{2}}\right)\right],
\end{aligned}
$$

we neglected $\mathcal{O}\left\{(D-4)^{2}\right\}$ contributions. Finally, we recall the familiar result,

$$
\Gamma\left(1-\frac{D}{2}\right)=\frac{2}{D-4}+\gamma_{\mathrm{E}}-1+\mathcal{O}(D-4)
$$

where $\gamma_{\mathrm{E}}$ is the Euler-Mascheroni constant. We thus arrive at

$$
\begin{aligned}
\Gamma_{1}=\int \mathrm{d}^{D} x & \frac{\sqrt{-g}}{4 \pi^{2}}\left\{( | c | ^ { 2 } - | d | ^ { 2 } ) \mu ^ { D - 4 } \left[\left(\frac{1}{2} m^{2} H^{2}(1-\epsilon)^{2}+\frac{1}{4} m^{4}\right)\right.\right. \\
& \times\left(\frac{2}{D-4}+\gamma_{\mathrm{E}}-1+\ln \left[\frac{H^{2}(1-\epsilon)^{2}}{2 \pi \mu^{2}}\right]\right) \\
& \left.+\int^{m} \mathrm{~d} \tilde{m}\left(\tilde{m}^{3}+\tilde{m} H^{2}(1-\epsilon)^{2}\right)[\psi(2-\mathrm{i} \tilde{\zeta})+\psi(2+\mathrm{i} \tilde{\zeta})]\right] \\
& \left.+2 H^{3}(1-\epsilon)^{3} \operatorname{Im}\left(c d^{*}\right) \int^{m} \mathrm{~d} \tilde{m} \Gamma(2+\mathrm{i} \tilde{\zeta}) \Gamma(2-\mathrm{i} \tilde{\zeta})\right\}
\end{aligned}
$$

Here, $\operatorname{Im}\left(c d^{*}\right)=|c||d| \sin \left(\phi_{c}-\phi_{d}\right)$ denotes the imaginary part of $c d^{*}$.

The one-loop backreaction contains a divergence when $D=4$. However, appreciate that the contribution to the effective action multiplying the mixed coefficients is finite in $D=4$. 


\subsection{Tree-level Friedmann equations}

Anticipating the renormalization procedure in the following section, we need the tree level, i.e. classical, Friedmann equations of motion responsible for driving the expansion of the universe. The system of interest is thus given by

$$
\begin{aligned}
S=\frac{1}{\kappa} \int \mathrm{d}^{D} x & \sqrt{-g}\{R-(D-2) \Lambda\}+\int \mathrm{d}^{D} x \sqrt{-g}\left\{-\frac{1}{2} g^{\mu \nu} \partial_{\mu} \varphi \partial_{\nu} \varphi-V(\varphi)\right\} \\
& +\int \mathrm{d}^{D} x \sqrt{-g}\left\{-\frac{1}{2} g^{\mu \nu} \partial_{\mu} \phi \partial_{\nu} \phi-V(\phi)-g_{\mathrm{Y}} \bar{\psi} \psi \phi\right\} \\
& +\int \mathrm{d}^{D} x \sqrt{-g}\left\{\frac{\mathrm{i}}{2}\left[\bar{\psi} \gamma^{\mu} \nabla_{\mu} \psi-\left(\nabla_{\mu} \bar{\psi}\right) \gamma^{\mu} \psi\right]\right\} .
\end{aligned}
$$

Here, $\kappa=16 \pi \mathrm{G}_{\mathrm{N}}$ represents the rescaled Newton constant, and $\Lambda$ denotes the cosmological constant. The reader can easily recognize the usual Einstein-Hilbert action on the first line. We introduce a new scalar field $\varphi$, responsible for the dynamics of the universe. By an appropriate choice of the potential $V(\varphi)$, a scalar field can mimic any mixture of fluids relevant for the evolution of our universe (see, e.g., [55]). The last two lines in equation (99) are identical to (12) and contain the fermion field and the scalar field $\phi$ generating the mass of the fermion through a Yukawa coupling, as in (13). We write

$$
\begin{aligned}
& \varphi(x)=\varphi_{0}(t)+\delta \varphi(x) \\
& \phi(x)=\phi_{0}(t)+\delta \phi(x),
\end{aligned}
$$

where we assume the background fields $\varphi_{0}(t)$ and $\phi_{0}(t)$ to be homogeneous. Moreover, we assume that $\rho_{\varphi} \gg \rho_{\phi}$, such that $\varphi(x)$ drives the dynamics of the universe as stated above. The classical Friedmann equations of motion are

$$
\begin{aligned}
& H^{2}-\frac{1}{D-1} \Lambda-\frac{\kappa\left(\frac{1}{2} \dot{\varphi}_{0}^{2}+V\left(\varphi_{0}\right)\right)}{(D-1)(D-2)}=0 \\
& \dot{H}+\frac{D-1}{2} H^{2}-\frac{\Lambda}{2}+\frac{\kappa\left(\frac{1}{2} \dot{\varphi}_{0}^{2}-V\left(\varphi_{0}\right)\right)}{2(D-2)}=0,
\end{aligned}
$$

and moreover, the classical scalar field equation of motion reads

$$
\ddot{\varphi}_{0}+(D-1) H \dot{\varphi}_{0}+\frac{\partial V}{\partial \varphi}\left(\varphi_{0}\right)=0 .
$$

We can thus derive the following identities:

$$
\begin{aligned}
& \sqrt{\kappa} \dot{\varphi}_{0}=\sqrt{2(D-2) \epsilon} H \\
& \sqrt{\kappa} \frac{\partial V}{\partial \varphi}\left(\varphi_{0}\right)=-\sqrt{2(D-2) \epsilon}(D-1-\epsilon) H^{2} \\
& \sqrt{\kappa} \ddot{\varphi}_{0}=-\sqrt{2(D-2) \epsilon} \epsilon H^{2} \\
& \frac{\partial^{2} V}{\partial \varphi^{2}}\left(\varphi_{0}\right)=2(D-1-\epsilon) \epsilon H^{2} .
\end{aligned}
$$

Here, we have used assumption (1a). 


\subsection{Renormalization}

In order to derive equation (98), we have assumed a constant deceleration $\epsilon$, as in equation $(1 a)$. We now promote this constant to a dynamical quantity:

$$
\epsilon \rightarrow \epsilon(t) \text {. }
$$

We will motivate this step shortly. The effective action (98) now contains divergences in both $H(t)$ and $\epsilon(t)$ that we ought to cancel by an appropriate counterterm action. It is not possible to identify local covariant counterterms of curvature invariants only that remove the singularities in both of these quantities at the level of the effective action. We can use the tree-level equations of motion derived in (102) to renormalize the effective action, which is first order in $\hbar$. By making use of an infinitesimal field redefinition, one can show the following: substituting the zeroth-order equation of motion into the first-order contribution to the action yields, up to one loop, the same equation of motion as directly varying the action. We thus add the following counterterm action:

$\Gamma_{\mathrm{ct}}=\int \mathrm{d}^{D} x \sqrt{-g}\left(c_{1} \phi^{2} R+c_{2} \phi^{2} \frac{\partial^{2} V}{\partial \varphi^{2}}\left(\varphi_{0}\right)-c_{3} \kappa \phi^{2} g^{\mu \nu} \partial_{\mu} \varphi \partial_{\nu} \varphi+c_{4} \phi^{4}\right)$.

The form of the counterterm action is unique, which can be shown by making use of dimensional analysis and by requiring that only two time derivatives can act on $\varphi_{0}(t)$ or $a(t)$ for stability. These conditions limit us to the counterterms above and possibly $\phi^{2} \varphi \square \varphi$. However, one can easily verify that the latter term does not have the correct form required to cancel the divergences in (98).

We recall relation (13). We need to expand the various terms in the counterterm action above around $D=4$, by making use of $R=(D-1)(D-2 \epsilon) H^{2}$ and equation (102):

$$
\begin{aligned}
& R \simeq 6(2-\epsilon) H^{2}+(D-4)(7-2 \epsilon) H^{2} \\
& \frac{\partial^{2} V}{\partial \varphi^{2}}\left(\varphi_{0}\right) \simeq 2(3-\epsilon) \epsilon H^{2}+(D-4) 2 \epsilon H^{2} \\
& \kappa g^{\mu \nu} \partial_{\mu} \varphi \partial_{\nu} \varphi \simeq-4 \epsilon H^{2}-(D-4) 2 \epsilon H^{2},
\end{aligned}
$$

where we neglected all $\mathcal{O}\left\{(D-4)^{2}\right\}$ contributions in the equations above. We can now easily solve for the coefficients in the counterterm action:

$$
\begin{aligned}
& c_{1}=-g_{\mathrm{Y}}^{2} \frac{|c|^{2}-|d|^{2}}{48 \pi^{2}} \frac{\mu^{D-4}}{D-4}+c_{1}^{\mathrm{f}} \\
& c_{2}=g_{\mathrm{Y}}^{2} \frac{|c|^{2}-|d|^{2}}{8 \pi^{2}} \frac{\mu^{D-4}}{D-4}+c_{2}^{\mathrm{f}} \\
& c_{3}=-3 g_{\mathrm{Y}}^{2} \frac{|c|^{2}-|d|^{2}}{32 \pi^{2}} \frac{\mu^{D-4}}{D-4}+c_{3}^{\mathrm{f}} \\
& c_{4}=-g_{\mathrm{Y}}^{2} \frac{|c|^{2}-|d|^{2}}{8 \pi^{2}} \frac{\mu^{D-4}}{D-4}+c_{4}^{\mathrm{f}},
\end{aligned}
$$

where the divergent coefficients in $D=4$ are fixed to cancel the divergences occurring in (98), and where, e.g., $c_{1}^{\mathrm{f}}$ is a finite but arbitrary coefficient of the counterterm action. The renormalized one-loop effective action thus reads

$$
\Gamma_{1, \text { ren }}=\int \mathrm{d}^{4} x \frac{\sqrt{-g}}{4 \pi^{2}}\left\{( | c | ^ { 2 } - | d | ^ { 2 } ) \left[\left(\frac{1}{2} m^{2} H^{2}(1-\epsilon)^{2}+\frac{1}{4} m^{4}\right)\left(\gamma_{\mathrm{E}}-1+\ln \left[\frac{H^{2}(1-\epsilon)^{2}}{2 \pi \mu^{2}}\right]\right)\right.\right.
$$




$$
\begin{aligned}
& \left.-\frac{1}{12} m^{2} H^{2}(7-5 \epsilon)+\int^{m} \mathrm{~d} \tilde{m}\left(\tilde{m} H^{2}(1-\epsilon)^{2}+\tilde{m}^{3}\right)[\psi(2-\mathrm{i} \tilde{\zeta})+\psi(2+\mathrm{i} \tilde{\zeta})]\right] \\
& \left.+2 H^{3}(1-\epsilon)^{3} \operatorname{Im}\left(c d^{*}\right) \int^{m} \mathrm{~d} \tilde{m} \Gamma(2+\mathrm{i} \tilde{\zeta}) \Gamma(2-\mathrm{i} \tilde{\zeta})\right\} \\
& +\int \mathrm{d}^{4} x \sqrt{-g}\left(c_{1}^{\mathrm{f}} \phi^{2} R+c_{2}^{\mathrm{f}} \phi^{2} \frac{\partial^{2} V}{\partial \varphi^{2}}\left(\varphi_{0}\right)-c_{3}^{\mathrm{f}} \kappa \phi^{2} g^{\mu \nu} \partial_{\mu} \varphi \partial_{\nu} \varphi+c_{4}^{\mathrm{f}} \phi^{4}\right)
\end{aligned}
$$

The effective action above comprises the one-loop backreaction of fermions. This term gives rise to additional physical corrections to the (classical) Friedmann equations, thus obtaining the so-called quantum corrected Friedmann equations. We will study its effect on the evolution of our universe and its impact on the evolution of the scalar field to which the fermions are coupled in a future publication. In this equation, we have neglected all contributions to the one-loop effective action arising from the trace anomaly.

If we expand equation (107) to first order in $\epsilon$, we can find out how large the first-order correction to the de Sitter result is. We have

$$
\begin{aligned}
\Gamma_{1, \text { ren }}=\Gamma_{1, \text { ren }}^{\Lambda} & +\epsilon \int \mathrm{d}^{4} x \frac{\sqrt{-g}}{4 \pi^{2}}\left\{( | c | ^ { 2 } - | d | ^ { 2 } ) \left[m^{2} H^{2}\left\{\frac{5}{12}-\gamma_{\mathrm{E}}-\ln \left[\frac{H^{2}}{2 \pi \mu^{2}}\right]\right\}-\frac{1}{2} m^{4}\right.\right. \\
& +\int^{m} \mathrm{~d} \tilde{m} \mathrm{i}\left(\tilde{m}^{2} H+\frac{\tilde{m}^{4}}{H}\right)\left[\psi^{\prime}(2+\mathrm{i} \tilde{m} / H)-\psi^{\prime}(2-\mathrm{i} \tilde{m} / H)\right] \\
& \left.-2 \tilde{m} H^{2}[\psi(2+\mathrm{i} \tilde{m} / H)+\psi(2-\mathrm{i} \tilde{m} / H)]\right] \\
& +2 H^{3} \operatorname{Im}\left(c d^{*}\right) \int^{m} \mathrm{~d} \tilde{m} \Gamma(2+\mathrm{i} \tilde{m} / H) \Gamma(2-i \tilde{m} / H) \\
& \left.\times\left[\mathrm{i} \frac{\tilde{m}}{H}(\psi(2+\mathrm{i} \tilde{m} / H)-\psi(2-\mathrm{i} \tilde{m} / H))-3\right]\right\}+\mathcal{O}\left(\epsilon^{2}\right)
\end{aligned}
$$

where the prime denotes a derivative with respect to the argument, and the superscript $\Lambda$ represent the well-known de Sitter result, which trivially follows from (107) by letting $\epsilon \rightarrow 0$.

Let us at this point however already consider the $\zeta \gg 1$ limit, to extract the physical behaviour of the effective potential resulting from (107). Note firstly that

$$
\Gamma_{1, \text { ren }}=\int \mathrm{d}^{4} x \sqrt{-g}\left(-V_{\text {eff }}(\phi)\right)
$$

where $V_{\text {eff }}(\phi)$ denotes the one-loop effective potential for $\phi$. In order to evaluate the integrals over $\tilde{m}$ occurring in equation (107), we need the following asymptotic expansions $(\zeta \in \mathbb{R})$ :

$$
\lim _{\zeta \rightarrow \infty} \Gamma(2+\mathrm{i} \zeta) \Gamma(2-\mathrm{i} \zeta)=2 \pi|\zeta|^{3} \mathrm{e}^{-\pi|\zeta|}\left(1+\frac{1}{\zeta^{2}}+\mathcal{O}\left(\frac{1}{\zeta^{4}}\right)\right)
$$

and also

$$
\lim _{\zeta \rightarrow \infty}[\psi(2+i \zeta)+\psi(2-i \zeta)]=\ln \left[\zeta^{2}\right]+\frac{13}{6} \frac{1}{\zeta^{2}}-\frac{119}{60} \frac{1}{\zeta^{4}}+\mathcal{O}\left(\frac{1}{\zeta^{6}}\right)
$$

Keeping the leading-order terms of the effective potential (109) in this limit only, we find from (107) and (110-111)

$$
V_{\mathrm{eff}}(\phi) \rightarrow-\frac{|c|^{2}-|d|^{2}}{16 \pi^{2}} g_{\mathrm{Y}}^{4} \phi^{4} \ln \left[\frac{g_{\mathrm{Y}}^{2} \phi^{2}}{2 \pi \mu^{2}}\right]+\frac{\operatorname{Im}\left(c d^{*}\right)}{\pi^{2}} g_{\mathrm{Y}}^{3} \phi^{3} H(1-\epsilon) \exp \left[-\pi \frac{g_{\mathrm{Y}}|\phi|}{H|1-\epsilon|}\right]
$$


where equation (13) has been used. Without studying the dynamics resulting from this asymptotic effective potential, let us briefly consider its stability properties. Clearly, this potential is unstable in the vacuum $(|d|=0)$ because the effective potential is unbounded from below in this case. This wild behaviour has already been recognized in de Sitter spacetime [19].

An interesting phenomenon can be observed precisely when $|c|^{2}=|d|^{2}=1 / 2$. This is realized, for example, in a thermal state with a temperature $T$ much larger than the UV cutoff of the theory. In this case, the asymptotic effective potential is exponentially suppressed and its (small) contributions can either be positive or negative.

\subsection{Discussion of the renormalized effective action}

Let us make a few general remarks. Consider quantum field theories in de Sitter spacetime. The Hubble parameter $H$ in de Sitter spacetime is a constant. An effective action for fermions or scalars in de Sitter spacetime contains divergences, for example, proportional to a power of $H$ (see, e.g., $[15,19,36,54])$. The same statement holds for divergences in the stress-energy tensor in, for example, the trace anomaly literature (see, e.g., [25, 36]). At this point, one can interpret the results differently:

(1) The Hubble parameter can quite conservatively be treated as a constant. The argument for this case is simple: throughout the calculation one simply assumed a constant Hubble parameter.

(2) The Hubble parameter is promoted to a dynamical quantity:

$$
H \rightarrow H(t) \text {. }
$$

A reason for supporting this case would be the following: when $\epsilon \ll 1$ one would find the de Sitter result as a leading-order contribution in each time interval where the Hubble parameter varies only adiabatically slowly, a spacetime also known as locally de Sitter spacetime.

Depending on which of the two interpretations one adheres to, one would renormalize differently. If one advocates the first point of view, all terms of, e.g., an effective action merely contribute to the renormalized cosmological constant. If, however, one prefers the second interpretation, one adds (dynamical) curvature invariants in order to cancel these divergences in a covariant manner.

We observe the following. In the effective action (107), the Hubble parameter is a dynamical quantity. Hence, covariant counterterms added to cancel UV divergences can and should be treated dynamically. This allows us to study the backreaction of fermions in a dynamical manner.

Albeit strictly speaking not allowed to relax the assumption $H=$ const in de Sitter spacetime, we conclude that promoting the Hubble parameter to a dynamical quantity $H=H(t)$ has been a useful approach. When in our calculation we keep $\epsilon$ constant as assumed, result (93) favours the second interpretation above.

Let us now return to our calculation to see what the above considerations can tell us about our effective action (93). We face the following two options:

(1) We treat $\epsilon$ as a constant.

(2) We promote $\epsilon$ to a dynamical quantity, as already stated in (103). We could call this a spacetime with an almost constant deceleration.

Despite the fact that throughout the calculation we have assumed that $\epsilon$ is a constant, it will come as no surprise that we argue in favour of the latter option for two reasons: if one 
would allow $\epsilon$ to change, one would probably find our main result for the effective action before renormalization (93) in each time interval where $\epsilon$ changes only very slowly. Secondly, the latter option is the generalization of the confirmed de Sitter case above.

An obvious disadvantage of our choice is that we can only renormalize on shell. Consider the divergences in (98). As already mentioned, it is not possible to identify local covariant counterterms of curvature invariants only that remove all singularities in $H(t)$ and $\epsilon(t)$ at the level of the effective action. Therefore, we relied on an on-shell renormalization technique and inserted the zeroth-order (tree-level) Friedmann equations of motion into the effective action at first order in $\hbar$.

Finally, let us try to comfort the reader who would rather keep $\epsilon$ constant, but who would simultaneously like to understand how to improve on his final result for the renormalized effective action. In this case, the only appropriate counterterms to renormalize (98) available to us are the following:

$$
\Gamma_{\mathrm{ct}}^{\mathrm{alt}}=\sqrt{-g}\left(\alpha \phi^{2} R+\beta \phi^{4}\right) .
$$

We can then renormalize the theory as usual ${ }^{3}$, with

$$
\begin{aligned}
& \alpha=-g_{\mathrm{Y}}^{2} \frac{|c|^{2}-|d|^{2}}{24 \pi^{2}} \frac{\mu^{D-4}}{D-4} \frac{(1-\epsilon)^{2}}{2-\epsilon}+\alpha_{\mathrm{f}} \\
& \beta=-g_{\mathrm{Y}}^{4} \frac{|c|^{2}-|d|^{2}}{8 \pi^{2}} \frac{\mu^{D-4}}{D-4}+\beta_{\mathrm{f}} .
\end{aligned}
$$

We could now study mode mixing ${ }^{4}$. One could simply allow $\epsilon$ and $m / H$ to vary in time and choose (if necessary infinitesimally small) time intervals in which $\epsilon$ and $m / H$ vary only slowly. Subsequently, one can match the solutions in two neighbouring intervals and study mode mixing in the spirit of [56]. In this case, this procedure would imply that we would have to renormalize for each time interval separately, for the coefficients in equation (115) depend on $\epsilon$. This is not surprising: a sudden jump, even infinitesimally small, in, for example, $m / H$ is not a physical phenomenon and would call for non-local counterterms (see, e.g., [57]).

\section{Conclusion}

In this paper, we have constructed the fermionic propagator in realistic FLRW spacetimes with the constant deceleration parameter $q=\epsilon-1$. Moreover, we assumed that $m / H$ is also constant, which can be realized in Yukawa theory by means of a scalar field for which $\phi \propto H$. We have derived the propagator both in the (trivial) massless case (25) and in the massive case (78).

Two pillars support our derivation. We split the fermionic degrees of freedom into a direct product of chirality and helicity eigenspinors. We normalize the spinors using a consistent canonical quantization and we require orthogonality of particle and anti-particle spinors.

Moreover, we verify that upon sending $\epsilon$ to zero, we recover the known vacuum-to-vacuum de Sitter results in the literature.

3 The case $\epsilon=2$ would have to be considered separately, for $R=0$ in a radiation-dominated universe.

4 As already formulated in [56] a (numerical) study of mode mixing is the answer to the fundamental problem that we can only solve for the mode functions exactly for a limited number of choices of $a(t)$. In their paper, Tsamis and Woodard study mode mixing for a massless minimally coupled scalar field in constant $\epsilon$ FLRW spacetimes. The basic strategy is as follows: in each-possibly infinitesimally small—time interval $\epsilon$ can be well approximated by a constant. The mode functions in two adjacent time intervals can be related by imposing two conditions: (i) continuity of the mode functions and (ii) continuity of their first derivatives. Hence, the mode functions at some later time $\eta$ can be obtained from the initial condition at $\eta^{\prime}$ by means of a transfer matrix. 
We calculate the one-loop effective action induced by fermions using our propagator. The one-loop backreaction arises from integrating out a free, quadratic fermion field and, using dimensional regularization; this generates a correction to the (classical) Friedmann equations. This effective action allows for the first time for a dynamical interpretation of $H$ such that the study of dynamical backreaction either on the background spacetime or on scalar fields coupled to these fermions is within reach.

\section{Acknowledgments}

JFK thanks Gerben Stavenga, Shun-Pei Miao and Timothy Budd for useful suggestions. The authors thank Gerard 't Hooft, Gleb Arutyunov and Tomas Janssen and acknowledge financial support from FOM grant 07PR2522 and by Utrecht University.

\section{Appendix A. Properties of Hankel functions}

In section 4.1.4, we used the following well-known identities involving Hankel functions:

$$
\begin{aligned}
& H_{-v}^{(1)}(z)=\mathrm{e}^{\mathrm{i} \pi v} H_{v}^{(1)}(z) \\
& H_{-v}^{(2)}(z)=\mathrm{e}^{-\mathrm{i} \pi v} H_{v}^{(2)}(z) \\
& \left\{H_{v}^{(1)}(z)\right\}^{*}=H_{v^{*}}^{(2)}\left(z^{*}\right) \\
& H_{v}^{(1)}\left(\mathrm{e}^{\mathrm{i} \pi} z\right)=-H_{-v}^{(2)}(z)=-\mathrm{e}^{-\mathrm{i} \pi v} H_{v}^{(2)}(z) \\
& H_{v}^{(2)}\left(\mathrm{e}^{-\mathrm{i} \pi} z\right)=-H_{-v}^{(1)}(z)=-\mathrm{e}^{\mathrm{i} \pi v} H_{v}^{(1)}(z) .
\end{aligned}
$$

The Wronskian of two Hankel functions reads

$$
W\left[H_{v}^{(1)}(z), H_{v}^{(2)}(z)\right]=-\frac{4 \mathrm{i}}{\pi z} .
$$

Moreover, we made use of the following recurrence relation:

$$
H_{v-1}^{(i)}(z)=\frac{\mathrm{d}}{\mathrm{d} z} H_{v}^{(i)}(z)+\frac{v}{n z} H_{v}^{(i)}(z) .
$$

\section{Appendix B. Fourier transforming Hankel functions}

Consider the following integral equation:

$$
I \equiv \int_{0}^{\infty} \mathrm{d} x x^{\mu+1} J_{\mu}(c x) K_{v}(a x) K_{v}(b x)
$$

Here, $J_{\mu}(z)$ is a Bessel function of the first kind, and $K_{v}(z)$ is a MacDonald function. Note that for all integrals in (76) both $a$ and $b$ are purely imaginary. The requirements from [46] are such that

$$
\begin{aligned}
& \operatorname{Re}(a)>0 \\
& \operatorname{Re}(b)>0 \\
& c>0 \\
& \operatorname{Re}(\mu \pm v)>-1 \\
& \operatorname{Re}(\mu)>-1 .
\end{aligned}
$$


Hence we introduce small real contributions in the arguments of both MacDonald functions only to take these to zero again at the end of the calculation. We thus write

$$
\begin{aligned}
& a=\delta+\mathrm{i} \alpha \\
& b=\delta+\mathrm{i} \beta .
\end{aligned}
$$

We choose $\delta>0$ to make the integral convergent. We now use equation (6.578.10) from [46] to find

$$
I=\frac{\sqrt{\pi} c^{\mu} \Gamma(\mu+v+1) \Gamma(\mu-v+1)}{2^{3 / 2}(a b)^{\mu+1}\left(u^{2}-1\right)^{\frac{1}{2} \mu+\frac{1}{4}}} P_{\nu-\frac{1}{2}}^{-\mu-\frac{1}{2}}(u)
$$

where

$$
u=\frac{a^{2}+b^{2}+c^{2}}{2 a b}
$$

Here, $P_{\nu-\frac{1}{2}}^{-\mu-\frac{1}{2}}(u)$ represents the associated Legendre function which we can rewrite in terms of a Gauss' hypergeometric function ${ }_{2} F_{1}$ by means of equations (8.702) and (9.131.1) of [46]: $I=\frac{\sqrt{\pi}(c / 2)^{\mu} \Gamma(\mu+v+1) \Gamma(\mu-v+1)}{4(a b)^{\mu+1} \Gamma(\mu+3 / 2)}{ }_{2} F_{1}\left(\mu+v+1, \mu-v+1 ; \mu+\frac{3}{2} ; \frac{1-u}{2}\right)$.

Upon taking $\delta \rightarrow 0$ we find

$$
\lim _{\delta \rightarrow 0} \frac{1}{(a b)^{\mu+1}}=\frac{1}{|a b|^{\mu+1}} \exp [-\mathrm{i} \pi(\mu+1) \theta(\alpha \beta) \operatorname{sgn}(\alpha+\beta)] .
$$

Finally, note that we can further simplify the argument of the hypergeometric function in equation (B.6) to

$$
\frac{1-u}{2}=1+\frac{c^{2}-(\alpha+\beta+\mathrm{i} \delta)^{2}}{4 \alpha \beta}
$$

Let us finally stress that we have introduced $\delta>0$ to make the integral convergent. It is, however, of significant physical importance for it is the same $\delta$ that dictates the $\varepsilon$ pole prescription in the propagator (see equations (78)-(79) and (22)). This should not come as a surprise: we observe the same behaviour in, e.g., Minkowski and de Sitter spacetimes.

\section{References}

[1] Ford L H 1985 Quantum instability of de Sitter space-time Phys. Rev. D 31710

[2] Antoniadis I and Mottola E 1991 Graviton fluctuations in de Sitter space J. Math. Phys. 321037

[3] Tsamis N C and Woodard R P 1994 The structure of perturbative quantum gravity on a de Sitter background Commun. Math. Phys. 162217

[4] Tsamis N C and Woodard R P 1996 Quantum gravity slows inflation Nucl. Phys. B 474235 (arXiv:hep-ph/9602315)

[5] Tsamis N C and Woodard R P 1996 One loop graviton self-energy in a locally de Sitter background Phys. Rev. D 542621 (arXiv:hep-ph/9602317)

[6] Abramo L R W, Brandenberger R H and Mukhanov V F 1997 The energy-momentum tensor for cosmological perturbations Phys. Rev. D 563248 (arXiv:gr-qc/9704037)

[7] Finelli F, Marozzi G, Vacca G P and Venturi G 2005 Adiabatic regularization of the graviton stress-energy tensor in de Sitter space-time Phys. Rev. D 71023522 (arXiv:gr-qc/0407101)

[8] Prokopec T 2006 A solution to the cosmological constant problem arXiv:gr-qc/0603088

[9] Prokopec T, Tsamis N C and Woodard R P 2008 Stochastic inflationary scalar electrodynamics Ann. Phys. 3231324 (arXiv:0707.0847 [gr-qc]) 
[10] Bilandzic A and Prokopec T 2007 Quantum radiative corrections to slow-roll inflation Phys. Rev. D 76103507 (arXiv:0704.1905 [astro-ph])

[11] Janssen T and Prokopec T 2008 A graviton propagator for inflation Class. Quant. Grav. 25055007 (arXiv:0707.3919 [gr-qc])

[12] Janssen T, Miao S P and Prokopec T 2008 Graviton one-loop effective action and inflationary dynamics arXiv:0807.0439 [gr-qc]

[13] Janssen T and Prokopec T 2008 Implications of the graviton one-loop effective action on the dynamics of the universe arXiv:0807.0447 [gr-qc]

[14] Janssen T M, Miao S P, Prokopec T and Woodard R P 2008 Infrared propagator corrections for constant deceleration Class. Quant. Grav. 25245013 (arXiv:0808.2449 [gr-qc])

[15] Candelas P and Raine D J 1975 General relativistic quantum field theory-an exactly soluble model Phys. Rev. D 12965

[16] Allen B and Lutken C A 1986 Spinor two point functions in maximally symmetric spaces Commun. Math. Phys. 106201

[17] Camporesi R 1992 The spinor heat kernel in maximally symmetric spaces Commun. Math. Phys. 148283

[18] Miao S P and Woodard R P 2006 The fermion self-energy during inflation Class. Quant. Grav. 231721 (arXiv:gr-qc/0511140)

[19] Miao S P and Woodard R P 2006 Leading log solution for inflationary yukawa Phys. Rev. D 74044019 (arXiv:gr-qc/0602110)

[20] Tomboulis E T 1990 Dynamically adjusted cosmological constant and conformal anomalies Nucl. Phys. B 329410

[21] Antoniadis I and Mottola E 1992 4-D quantum gravity in the conformal sector Phys. Rev. D 452013

[22] Antoniadis I 1992 Dynamics of the conformal factor in 4-D gravity arXiv:hep-th/9211055

[23] Antoniadis I, Mazur P O and Mottola E 1998 Fractal geometry of quantum spacetime at large scales Phys. Lett. B 444284 (arXiv:hep-th/9808070)

[24] Salehi H and Bisabr Y 2000 Conformal anomaly and large scale gravitational coupling Int. J. Theor. Phys. 391241 (arXiv:hep-th/0001095)

[25] Antoniadis I, Mazur P O and Mottola E 2007 Cosmological dark energy: prospects for a dynamical theory New J. Phys. 911 (arXiv:gr-qc/0612068)

[26] Koksma J F and Prokopec T 2008 Effect of the trace anomaly on the cosmological constant Phys. Rev. D 78023508 (arXiv:0803.4000 [gr-qc])

[27] Chernikov N A and Tagirov E A 1968 Quantum theory of scalar fields in de Sitter space-time Ann. Poincare Phys. Theor. A 9109

[28] Allen B 1985 Vacuum states in de Sitter space Phys. Rev. D 323136

[29] Allen B and Folacci A 1987 The massless minimally coupled scalar field in de Sitter space Phys. Rev. D 353771

[30] Allen B and Jacobson T 1986 Vector two point functions in maximally symmetric spaces Commun. Math. Phys. 103669

[31] Tsamis N C and Woodard R P 2007 A maximally symmetric vector propagator J. Math. Phys. 48052306 (arXiv:gr-qc/0608069)

[32] Allen B 1986 The graviton propagator in de Sitter space Phys. Rev. D 343670

[33] Allen B and Turyn M 1987 An evaluation of the graviton propagator in de Sitter space Nucl. Phys. B 292813

[34] Floratos E G, Iliopoulos J and Tomaras T N 1987 Tree level scattering amplitudes in de Sitter space diverge Phys. Lett. B 197373

[35] Higuchi A and Kouris S S 2001 The covariant graviton propagator in de Sitter spacetime Class. Quant. Grav. 184317 (arXiv:gr-qc/0107036)

[36] Birrell N D and Davies P C W 1982 Quantum fields in curved space Cambridge monographs on Mathematical Physics (Cambridge: Cambridge University Press)

[37] Prokopec T and Puchwein E 2004 Photon mass generation during inflation: de Sitter invariant case J. Cosmol. Astropart. Phys. JCAP04(2004)007 (arXiv:astro-ph/0312274)

[38] Weinberg S 2005 Quantum contributions to cosmological correlations Phys. Rev. D 72043514 (arXiv:hep-th/0506236)

[39] Koksma J F, Prokopec T and Rigopoulos G I 2008 The scalar field kernel in cosmological spaces Class. Quant. Grav. 25125009 (arXiv:0712.3685 [gr-qc])

[40] Cotaescu I I 2002 Polarized Dirac fermions in de Sitter spacetime Phys. Rev. D 65084008 (arXiv:hep-th/0109199)

[41] Garbrecht B and Prokopec T 2006 Fermion mass generation in de Sitter space Phys. Rev. D 73064036 (arXiv:gr-qc/0602011)

[42] De Wit B and Smith J 1986 Field Theory in Particle Physics vol 1 (Amsterdam: North-Holland Personal Library) 
[43] Peskin M E and Schroeder D V 1995 An Introduction to Quantum Field Theory (Reading, MA: Addison-Wesley)

[44] Itzykson C and Zuber J B 1980 Quantum Field Theory (New York: Mcgraw-hill)

[45] Prokopec T, Schmidt M G and Weinstock S 2004 Transport equations for chiral fermions to order h-bar and electroweak baryogenesis Ann. Phys. 314208 (arXiv:hep-ph/0312110)

[46] Gradshteyn I S and Ryzhik I M 1965 Table of Integrals, Series, and Products (New York: Academic)

[47] Prudnikov A P, Brychkov Yu A and Marichev O I 1986 Special functions Integrals and Series vol 2 (London: Gordon and Breach Science )

[48] Mottola E 1985 Particle creation in de Sitter space Phys. Rev. D 31754

[49] Goldstein K and Lowe D A 2003 A note on alpha-vacua and interacting field theory in de Sitter space Nucl. Phys. B 669325 (arXiv:hep-th/0302050)

[50] Einhorn M B and Larsen F 2003 Squeezed states in the de Sitter vacuum Phys. Rev. D 68064002 (arXiv:hep-th/0305056)

[51] Janssen T M, Miao S P, Prokopec T and Woodard R P 2009 The hubble effective potential arXiv:0904.1151 [gr-qc)

[52] Janssen T M, Miao S P, Prokopec T and Woodard R P in preparation

[53] Inagaki T, Mukaigawa S and Muta T 1995 A soluble model of four fermion interactions in de Sitter space Phys. Rev. D 524267 (arXiv:hep-th/9505058)

[54] Inagaki T, Muta T and Odintsov S D 1997 Dynamical symmetry breaking in curved spacetime: four-fermion interactions Prog. Theor. Phys. Suppl. 12793 (arXiv:hep-th/9711084)

[55] Padmanabhan T 2002 Accelerated expansion of the universe driven by tachyonic matter Phys. Rev. D 66021301 (arXiv:hep-th/0204150)

[56] Tsamis N C and Woodard R P 2003 Plane waves in a general Robertson-Walker background Class. Quant. Grav. 205205 (arXiv:astro-ph/0206010)

[57] Vilenkin A and Ford L H 1982 Gravitational effects upon cosmological phase transitions Phys. Rev. D 261231 